\title{
Functional Organization for Direction of Motion and Its Relationship to Orientation Maps in Cat Area 18
}

\author{
Amir Shmuel and Amiram Grinvald \\ Department of Neurobiology, The Weizmann Institute of Science, Rehovot 76100, Israel
}

The goal of this study was to explore the functional organization of direction of motion in cat area 18. Optical imaging was used to record the activity of populations of neurons. We found a patchy distribution of cortical regions exhibiting preference for one direction over the opposite direction of motion. The degree of clustering according to preference of direction was two to four times smaller than that observed for orientation. In general, direction preference changed smoothly along the cortical surface; however, discontinuities in the direction maps were observed. These discontinuities formed lines that separated pairs of patches with preference for opposite directions. The functional maps for direction and for orientation preference were closely related; typically, an iso-orientation patch was divided into regions that exhibited preference for opposite directions, orthogonal to the orientation. In addition, the lines of disconti-

Since the early studies performed by Mountcastle (1957) and Hubel and Wiesel $(1962,1963)$, it has been known that in several areas of the cerebral cortex, cells that exhibit similar selectivity in their response properties are clustered together. Cells that are aligned vertically with respect to the surface of the cortex maintain the same type of selectivity for several features (for review, see LeVay and Nelson, 1991). This paper addresses the question of whether clustering for direction of motion (hereafter "direction") exists in cat area 18 . We also explore the possible relationship to the cortical layout for orientation selectivity.

In their pioneering research, Hubel and Wiesel (1965) showed that cells in area 18 present both sharp orientation tuning and direction selectivity. Nevertheless, although a columnar arrangement was reported for orientation preference, there was no indication for clustering of cells with similar direction preferences. Only later was such clustering reported in both area 18 (Payne et al., 1980) and area 17 (Tolhurst et al., 1981). Subsequently, Berman et al. (1987) questioned the possibility of a columnar organization for direction selectivity, from pia to the white matter. In both areas 17 and 18, direction preference along vertical tracks reverses at least once. Thus, direction selectivity may be a stimulus attribute that is not mapped in the classical columnar structure.

In tangential tracks, preferred direction and orientation change

Received March 19, 1996; revised July 18, 1996; accepted July 22, 1996.

This work was supported by The Israel Science Foundation, the Wolfson Foundation, and a grant from Ms. Margaret Enoch. We thank D. Malonek for introducing us to optical imaging of direction selectivity, A. Arieli for constructing the device for targeted electrical recording, and T. Bonhoeffer for conducting one experiment presented here with A.G. The contributions of A. Aertsen, D. Ettner, D. Glaser, S. Leytus, R. Malach, D. Shoham, E. Shtoyerman, I. Vanzetta, and C. Wijnbergen are gratefully acknowledged.

Correspondence should be addressed to Amir Shmuel, Department of Neurobiology, The Weizmann Institute of Science, Rehovot 76100, Israel.

Copyright (C) 1996 Society for Neuroscience $0270-6474 / 96 / 166945-20 \$ 05.00 / 0$ nuity within the direction map often connected points of singularity in the orientation map. Although the organization of both domains was related, the direction and the orientation selective responses were separable; whereas the selective response according to direction of motion was nearly independent of the length of bars used for visual stimulation, the selective response to orientation decreased significantly with decreasing length of the bars. Extensive single and multiunit electrical recordings, targeted to selected domains of the functional maps, confirmed the features revealed by optical imaging. We conclude that significant processing of direction of motion is performed early in the cat visual pathway.

Key words: cat; visual cortex; area 18; functional architecture; cortical map; direction selectivity; orientation selectivity; optical imaging; intrinsic signals

together in small increments (Berman et al., 1987). Occasionally, a large jump in preferred direction occurs with only a small change in preferred orientation. A model that projects the suggested functional architecture for direction selectivity on top of the functional architecture for the orientation domain has been proposed (Berman et al., 1987). The model is consistent with the "ice-cube" model of Hubel and Wiesel (1977) in terms of orientation. Preferred direction is orthogonal to preferred orientation. Each of the iso-orientation bands and each of the iso-orientation columns are divided into two sequences of preference for opposite direction.

An alternative model, supported by extensive electrical recordings, has been suggested by Swindale et al. (1987). Given that the orientation map is continuous, with the exception of the presence of $180^{\circ}$ singularities (Swindale 1982; Swindale et al., 1987; Bonhoeffer and Grinvald, 1991, 1993), and assuming that direction preference is always orthogonal to orientation preference, the map of direction preference cannot be continuous. The direction map, if it is assumed to be as continuous as possible, must nevertheless contain lines across which direction preference reverses by $180^{\circ}$. These lines begin and end in singularities within the orientation domain (Swindale et al., 1987).

Using the optical imaging technique based on intrinsic signals (Grinvald et al., 1986; Frostig et al., 1990; Malonek and Grinvald 1996; for review, see Bonhoeffer and Grinvald, 1996), Bonhoeffer and Grinvald (1993) presented inconclusive results regarding the clustering of direction-selective cells in cat area 18. Recent improvements of the signal-to-noise ratio obtained by optical imaging have allowed us to reexamine this question.

This study clearly establishes the functional organization for direction in cat area 18. We also provide a detailed description of 
the close relationship between the organization of direction and orientation preference.

A preliminary report has been published previously in abstract form (Shmuel et al., 1993).

\section{MATERIALS AND METHODS}

The methods used for preparing and maintaining the animals were described previously (Bonhoeffer and Grinvald, 1993). The methods are outlined below, whereas differences and new methodological aspects are described in detail.

\section{Animals}

Adult cats (10) and 8-week-old kittens (6) were used for this study. The methods used for adult cats were similar to those described previously by Bonhoeffer and Grinvald (1993). Kittens were anesthetized using halothane $/ \mathrm{N}_{2} \mathrm{O}$ anesthesia $\left(70 \% \mathrm{~N}_{2} \mathrm{O}, 30 \% \mathrm{O}_{2}\right.$ with $0.6-1.5 \%$ halothane). They were not paralyzed.

For both cats and kittens, atropine $(1 \%)$ was administered to the eyes to paralyze accommodation. A keratometer was used to fit the corneas with zero-power contact lenses. The eyes were focused on a tangent screen at a distance of $30-60 \mathrm{~cm}$ using appropriate external lenses, as determined by retinoscopy.

\section{Optical chamber}

The skull of the cat was opened above area 18 by drilling two semicircular holes whose "centers" lay at Horsley-Clark $\sim$ A4. The imaged area extended from $1 \mathrm{~mm}$ to $5 \mathrm{~mm}$ away from the midline. A stainless steel chamber was cemented onto the skull, the dura was removed, and the chamber was filled with silicon oil and sealed with a round glass cover. To facilitate electrical recordings through the sealed chamber, in some instances a rubber gasket was glued into a 3-mm-diameter hole in the round glass cover.

\section{CCD camera and optics}

Two different imaging systems were used for the imaging based on intrinsic signals. One system introduced by Ts'o et al. (1990) included a slow-scan CCD camera (Photometrics Ltd., Tuscon, AZ). The camera provided 12-bit digitized images of the cortex. These pictures had a spatial resolution of $192 \times 144$ pixels. The root-mean-square shot noise obtained by this camera configuration was 1 part in 1400 . The other imaging system used was a differential video data acquisition system, IMAGER 2001 (Optical Imaging, Germantown, NY). It included a video camera that provided a spatial resolution of $756 \times 574$ pixels. A reference image was subtracted from the video signal. The resulting signal was then amplified and digitized by an 8-bit image processor. This digitization of the differential-enhanced image was equivalent to 11-12 bit digitization. In addition to the increased spatial resolution obtained by this imaging system, the root-mean-square shot noise was approximately threefold better relative to that obtained by the CCD system. Moreover, the availability of the differential image in real time proved useful in assessing the noise level at an early stage.

The camera was mounted above the optical chamber and was aimed such that its optical axis was perpendicular to the cortical surface. To achieve nearly uniform illumination of the cortex, two adjustable light guides were aimed separately at the cortex. These guides were attached to a Zeiss tungsten-halogen lamp. The light was passed through interference filters of different wavelengths. The filter used for visualizing the surface of the cortex and its vascular pattern had a transmission maximum at $540 \pm 15 \mathrm{~nm}$. The filters used for the optical imaging had peak transmission wavelengths of 605,630 , and $650 \mathrm{~nm}$. The first filter was chosen because its maximum transmission wavelength coincides with the peak of the difference spectrum between oxyhemoglobin and deoxyhemoglobin. Use of this wavelength maximizes the contribution of oximetry signals relative to other intrinsic signals (Frostig et al., 1990). Whenever the slow vascular noise $(\sim 0.1 \mathrm{~Hz}$ slow oscillations $)$ seemed large relative to the respiratory signal, the two other filters were used to increase the contribution of the light-scattering component of the intrinsic signal (Frostig et al., 1990; Malonek and Grinvald, 1996). Evaluation of the slow noise level was made before the imaging session by inspecting the differential image in real time under green illumination.

To minimize artifacts caused by blood vessels on the surface of the cortex, a "tandem-lens" arrangement (macroscope) was used (Ratzlaff and Grinvald, 1991). The macroscope provided high numerical aperture.
Consequently, the optical system had a very shallow depth of field (nominal: $50 \mu \mathrm{m}$ ). Therefore, when focused $400 \mu \mathrm{m}$ below the cortical surface, the fine surface vasculature virtually disappeared.

\section{Visual stimuli}

We used a visual stimulator based on an IBM PC equipped with a graphics board (SGT, Number Nine Corporation, Lexington, MA). The software for this stimulator was developed by Kaare Christian, The Rockefeller University. The stimuli were displayed on a CRT screen in 60 $\mathrm{Hz}$ noninterlaced mode. The monitor was usually positioned at a distance of $30-60 \mathrm{~cm}$ from the animal, subtending an angle of $30-55^{\circ}$ in the visual field, contralateral to the hemisphere investigated. The cats were stimulated binocularly with high-contrast rectangular-wave gratings with a spatial frequency of $0.15-0.18$ cycles per degree and a temporal frequency of $2.5-6.0 \mathrm{~Hz}$. The duty cycle used for the grating pattern was in the range of $33-50 \%$. The main set of stimuli included eight differently oriented gratings, each of which moved in two opposite directions, orthogonal to the orientation. The set of stimuli spanned both the full orientation spectrum and the full direction spectrum at a resolution of $22.5^{\circ}$.

\section{Data acquisition}

For functional imaging, a particular stimulus was presented binocularly for $2.5 \mathrm{sec}$ while 10 frames of $500 \mathrm{msec}$ duration each were recorded. To allow for the relaxation of activity-dependent vascular changes, this period of data acquisition was followed by an $8 \mathrm{sec}$ interstimulus interval (ISI). An abrupt change in the pattern of visual stimulus produces a nonselective activation of the cortex. To minimize this effect, a stationary pattern of the following stimulus condition was displayed during the ISI. Subsequently, the pattern started to drift, and images of the cortex were taken. Data acquisition started $500 \mathrm{msec}$ before the onset of the stimulus motion.

Each of the different stimuli was presented once in each trial. The order of stimulus presentation in each trial was randomized to average out any systematic effects of stimulus presentation order. To reduce the noise in the acquired images, signal averaging was used, with each stimulus being presented 64-128 times. The data obtained during any 6-8 successive trials was summed to one block of data.

\section{Electrical recording}

Glass-coated tungsten microelectrodes were used, with a tip diameter of $15 \mu \mathrm{m}$ and impedance of $0.6 \mathrm{M} \Omega$, measured at $1 \mathrm{kHz}$. For recording along tangential tracks, the electrode was mounted on a hydraulic microdrive (Narishige MO 103; Narishige, Tokyo, Japan). Vertical penetrations were performed using a new design for targeted electrical recordings into optically imaged functional domains. Details of this apparatus will be described elsewhere (A. Arieli and A. Grinvald, unpublished observations).

For targeted electrical recording, the image of the cortical vasculature was superimposed onto the functional maps. The desired recording locations were marked on the functional maps and transferred automatically to the image of the cortical vasculature (see Fig. $6 F$ ). The electrode was manipulated to the desired recording site under visual control, while the cortical surface was viewed by means of an operating microscope. The fine pattern of superficial blood vessels was used to target the electrode to the proper location. The cortical depth for each recording was estimated by the total travel distance of the electrode after it first touched the cortex. The micromanipulation of the electrode in three dimensions was performed in the sealed recording chamber. The recordings from a sealed cranial chamber were stable over a long period of time.

The electrical signal was amplified and band-filtered at $300-3000 \mathrm{~Hz}$. This signal was transferred to both a spike sorter (MSD-2; Alpha-Omega, Nazareth, Israel) and a window discriminator. By using the spike sorter, it was usually possible to isolate one single unit; in some of the cortical locations, two single units were detected simultaneously. The window discriminator was used for recording multiunit activity. The outputs of both the window discriminator and the spike sorter were transferred to an IBM-PC-compatible computer, with which spikes were recorded using the Hist system (designed at the Rockefeller University and programmed by K. Christian).

\section{Analysis of functional maps}

Data analysis was performed using two image-analysis programs: TVmix (Optical Imaging) and MATLAB (The MATH WORKS) run on an 
IBM RISC System/6000 workstation. The first step in the data analysis was to sum the frames acquired for each visual stimulus. Next, functional maps were obtained by subtracting the baseline cortical image (details below). After this, several types of analyses were used for the presentations throughout this manuscript. These are described briefly here, with the details of the analyses described elsewhere (Ts'o et al., 1990; Bonhoeffer and Grinvald, 1993; Malonek et al., 1994; Bonhoeffer and Grinvald, 1996).

Single-condition maps. To obtain a single-condition map, the cortical image obtained while stimulating with the specific condition was divided by the "cocktail blank" cortical image. Cocktail blank refers to the sum of responses to all different stimuli. The next step in the analysis of functional maps included high-pass filtering, using an isotropic Gaussian whose standard deviation was equal to $500 \mu \mathrm{m}$ of cortical distance. This procedure was used to remove low spatial frequency noise in the image, thus avoiding distortion of the results (low-pass filtering was used only before the vectorial analysis). The result of the described procedure, a "single-condition map," is the map that represents the specific activity evoked by the specific condition. Dark patches in such a map are the areas that respond to this condition more than to the other ones.

The term "orientation single-condition map" refers to a functional map computed by taking the sum of the two cortical images obtained by stimulating with one grating pattern, moving in the two directions orthogonal to the orientation, and dividing by the cocktail blank. In the resulting map, the contributions of the direction-selective responses are mixed and thus canceled out. To compute an "orientation and direction singlecondition map," the cortical image obtained by stimulating with a given grating pattern moving in only one direction orthogonal to the orientation was divided by the cocktail blank. An orientation and direction single-condition map carries information regarding both the orientation of the stimulus and its direction. Thus, to isolate the direction-selective response, differential analysis is needed.

Differential maps. A differential map is the result of subtracting one single-condition map from another, each single-condition map being obtained using a different stimulus. Dark patches in a differential map represent areas preferentially activated by the first stimulus, whereas bright patches represent areas preferentially activated by the second. Differential direction maps are obtained by subtracting two orientation and direction single-condition maps of stimuli moving in opposite directions. Differential orientation maps are obtained by subtracting two orientation single-condition maps of gratings whose relative orientation is orthogonal.

Vectorial analysis. For comprehensive analysis of the organization of iso-direction domains, the responses to 16 different directions of motion of the grating stimuli were summed vectorially on a pixel-by-pixel basis (Blasdel and Salama, 1986; Ts'o et al., 1990). First, the single-condition activity maps were low-pass-filtered using an isotropic Gaussian whose standard deviation was equal to $70 \mu \mathrm{m}$ of cortical distance. Then, for every point in the cortex, 16 vectors were summed, their lengths being the magnitude of the single-condition responses and their angles corresponding to the direction of the gratings that produced the responses. An angle map presents the angle of the resulting vector by means of color coding for any piece of the cortex. The magnitude map presents the magnitude of the resulting vector. Note, however, that a vector with a low magnitude can be the result either of several stimulus directions, all evoking strong responses that cancel out, or a weak response to all directions. A polar map presents information about both preferred angle and magnitude of preference. The polar maps presented throughout this paper use either lines (for orientation) or arrows (for direction) to illustrate the local preference.

In addition to vectorial summation, we adopted a method proposed by Worgotter and Eysel (1987) to differentiate between direction selectivity and orientation selectivity. The essence of the technique is to apply a pixel-wise Fourier transform to the measured responses in the polar domain. The first component obtained in the frequency domain describes the preferred direction and the magnitude of preference. The second component describes the preferred orientation and the magnitude of orientation preference. Both methods for preference analysis, vectorial analysis and Fourier decomposition, gave nearly identical results.

Fracture analysis. To analyze the rate of change of the preferred orientation or direction, we applied a two-dimensional gradient operator to the corresponding angle map (see Appendix).

\section{Analysis of data obtained by electrical recording}

The set of stimuli for electrical recording was identical to that used for optical imaging. It was presented in a random order for five trials for each recording location. In the first stage of analysis, the number of spikes during the period of stimulus motion was summed over all trials for each type of stimulus. The result of this summation for each location or for each single cell is a vector of the responses to the different presented directions of motion. In the next stage, each of these vectors was normalized by dividing it by the sum of its components. The normalization prevents biases attributable to differences in the overall responsiveness of the various cells or recording sites. The resulting normalized vector is of the same type of pixel-by-pixel vectors of orientation and direction single-condition responses obtained by optical imaging. The analysis for preference of orientation and direction was identical to that performed for the optically recorded data.

\section{RESULTS}

\section{Similarity of activity patterns resulting from gratings moving in opposite directions}

To study the functional organization of area 18 with respect to direction, cats were presented with oriented gratings moving in directions that spanned the full direction spectrum $\left(360^{\circ}\right)$. The set of stimuli included eight differently oriented gratings, each of which was moved in two opposite directions, orthogonal to its orientation. Figure 1 illustrates 8 of the 16 orientation and direction single-condition maps obtained from the same cortical area. Each of these single-condition maps carries information regarding both the orientation and the direction of the stimulus. Black patches in each image represent cortical areas that were preferentially activated by gratings whose orientation and direction are marked on the image. Pairs of opposite images represent singlecondition maps obtained from gratings of the same orientation, moving in opposite directions. To facilitate the comparison of the activity maps comprising such a pair, white crosses are used to mark some of the patches that were activated by gratings of horizontal orientation. Similarly, two sets of black crosses are added to the activity maps obtained from vertical gratings. The functional maps obtained from gratings of the same orientation moving in opposite directions were similar but not identical. This result indicates that the clustering for orientation is prominent, whereas the clustering for direction is weak.

To quantitatively compare the various orientation and direction single-condition maps, we computed the correlation coefficients of all possible pairs of them. The results are shown in Figure $2 A$, in the format of a correlation matrix. Figure $2 B$ illustrates the summary of the results; the vector of correlation coefficients for every single-condition map with other single-condition maps is averaged over all single-condition maps. The averaged vector is presented as a function of the difference in direction corresponding to the maps. The average values confirmed quantitatively the aforementioned features of similarity between orientation and direction single-condition maps. Inspection of the full correlation matrix shown in Figure $2 A$ indicated that the trends of similarity that were quantified in Figure $2 B$ were not only true for the average of correlation coefficients but were valid for any individual single-condition map as well.

The similarity of functional maps obtained by gratings of the same orientation but opposite direction scored an average correlation coefficient of 0.60 . To assess the significance of this value, we compared it with the correlation coefficient obtained while testing the reproducibility of the single-condition maps. The average value of this correlation coefficient was higher: 0.82 . The comparison indicated that single-condition maps obtained from gratings of the same orientation moving in 

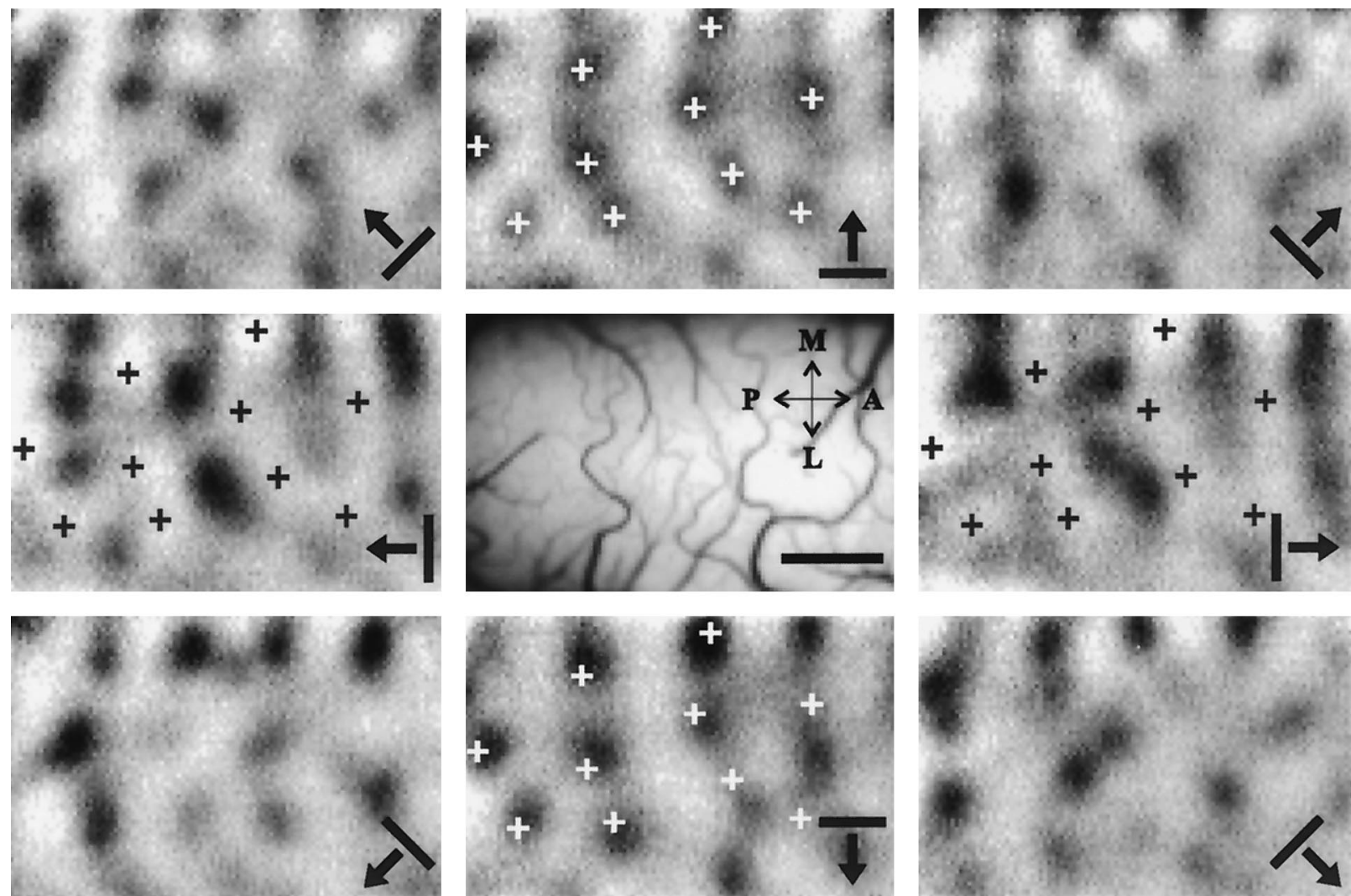

Figure 1. Activity patterns evoked by gratings moving in various directions. Eight different gratings, each moving in two opposite directions, were presented to the cat while activity maps were collected. A subset of eight orientation and direction single-condition optical maps is presented. Dark patches in each image represent cortical areas that were active while the cat was stimulated with gratings whose orientation and direction are marked on the image. The four sets of crosses are marked on identical cortical locations to enable easy comparison of the patterns of activity. The image in the center is the image of the cortical surface and superficial blood vessels, taken under green illumination $(540 \mathrm{~nm})$. Each of the different stimuli selectively activated a small number of patches in the imaged area. Maps produced by gratings of similar orientation, but opposite direction of motion (180 apart), were similar to one other. Gratings at orthogonal orientations activated complementary patches. The maps are scaled such that the whole range of gray levels corresponds to a fractional change of $3.2 \times 10^{-4}$ for presenting the activity maps. The wavelength of illumination used for imaging (hereafter wavelength) was $650 \pm$ $10 \mathrm{~nm}$. $A$, Anterior; $P$, posterior; $M$, medial; $L$, lateral. Scale bar, $1 \mathrm{~mm}$.
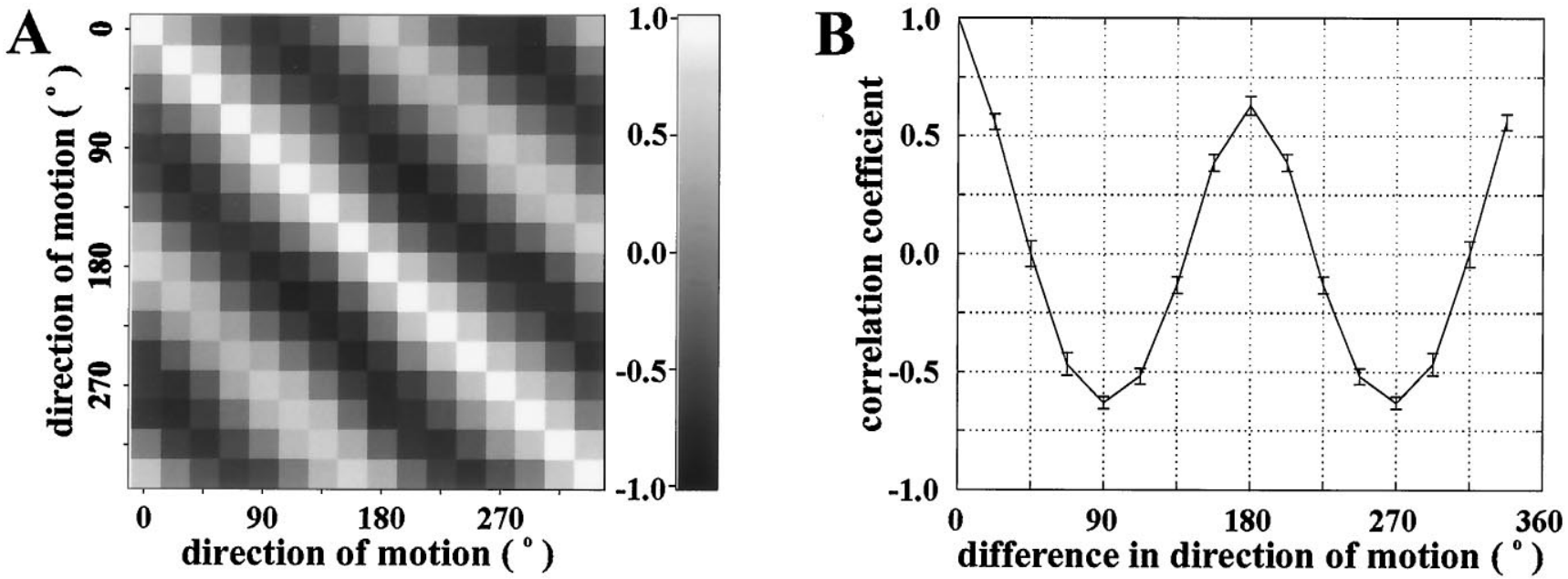

Figure 2. Correlations between single-condition maps. $A$, The features of similarity indicated for orientation and direction single-condition maps are demonstrated by the matrix of correlation coefficients among these activity maps. The comparison of each map to another was performed by calculating the correlation coefficient between corresponding pixels. The entry $(i, j)$ presents the correlation coefficient of the single-condition map corresponding to direction $(i-1) \pi / 8$ with that of the single-condition map corresponding to direction $(j-1) \pi / 8(i, j=1 \ldots 16)$. $B$, The observations of similarity are summarized in a correlation graph. The average correlation coefficient between pairs of single-condition maps is presented as a function of the angular difference in the direction corresponding to the compared maps. The average was computed by first cyclically shifting each row of the matrix in $A$ such that the entry of the diagonal was shifted to the first column, and then by averaging over the rows. Gratings of similar orientation that moved in opposite directions of motion produced similar maps $(r=0.60)$. Gratings of orthogonal orientation produced complementary maps $(r=-0.60)$. The average correlation between activity patterns produced by gratings whose relative orientation was oblique was $\sim 0$. 

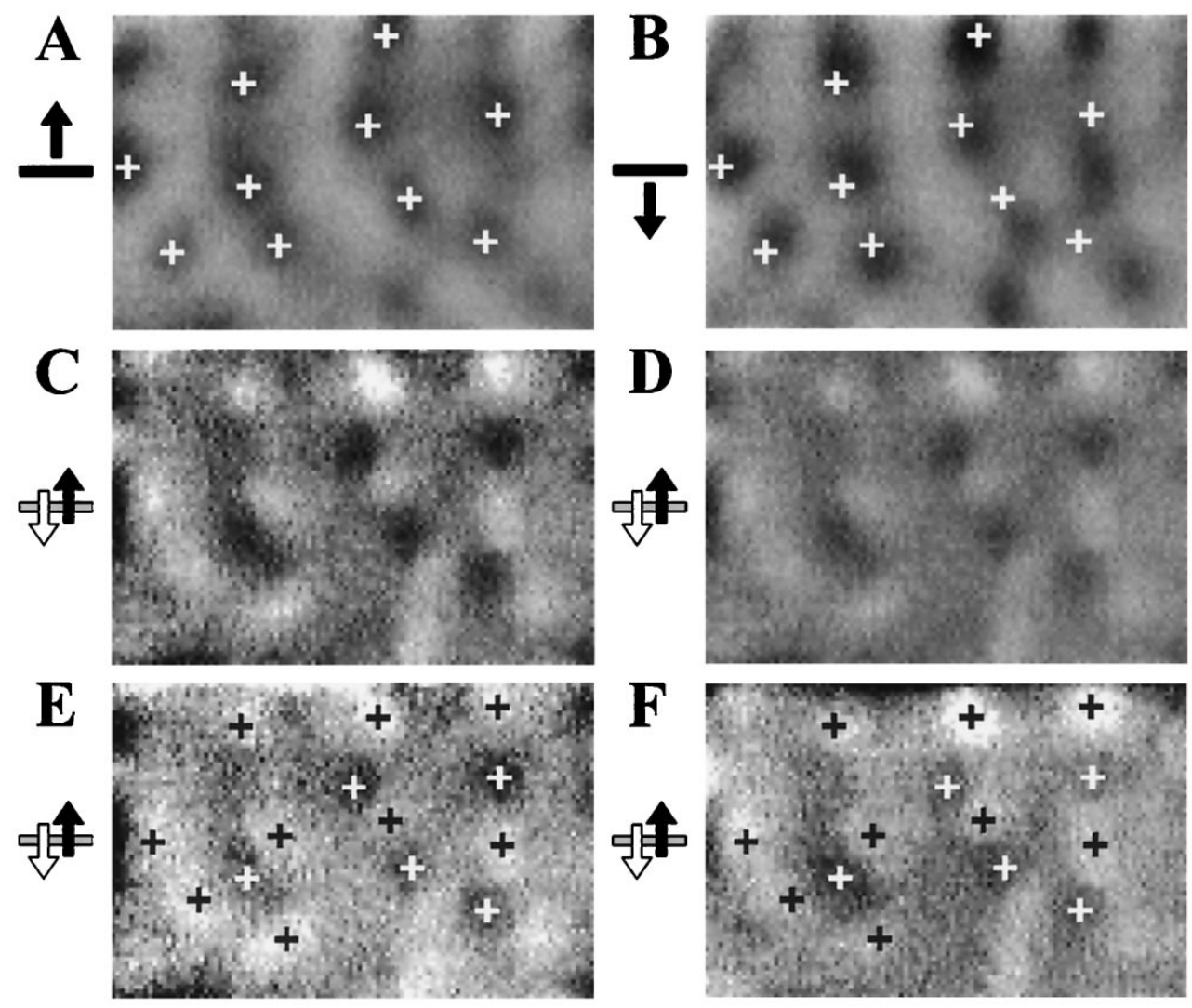

Figure 3. Clustering according to direction of motion in area 18. $A$ and $B$ illustrate orientation and direction singlecondition maps obtained from the same cortical area by stimulating with horizontal gratings moving upward and downward, respectively. The dark patches represent cortical areas activated by the stimulus marked next to the image. The entire range of gray levels represents a fractional change of $5 \times 10^{-4}$ (wavelength $650 \pm 10 \mathrm{~nm}$ ). The two sets of crosses are located in identical cortical locations. $C, \mathrm{~A}$ differential direction map between activity produced by a horizontal grating moving upward and a horizontal grating moving downward. The dark regions correspond to an upward direction preference and the bright regions to a downward direction preference. The map was computed by subtracting the single-condition map presented in $B$ from that presented in $A$. The differential direction map, of amplitude $2.9 \times 10^{-4}$, is scaled to the full range of gray levels. $D$, The same direction map as seen in $C$, presented in a scaled gray level whose entire range represents a fractional change of $5 \times 10^{-4}$. This scale was used to facilitate comparison of amplitudes with $A$ and $B . E$ and $F$ present the same direction map obtained from interlaced complementary blocks of data. The sets of crosses superimposed on both images correspond to identical cortical locations. Black and white crosses correspond to regions activated by downward and upward motion, respectively. The average S/N (signal-tonoise ratio; see Appendix for formal definition) of the eight direction maps in this experiment was $7.6 \pm 1.1$. The range of gray levels here represents a fractional change of $3.2 \times 10^{-4}$. Scale bar, $1 \mathrm{~mm}$. opposite directions contain additional information regarding features other than orientation.

\section{Clustering according to direction preference}

This result prompted us to examine whether functional maps for direction selectivity of relatively low amplitude do exist. To resolve this question directly, we canceled the effect of orientation on the functional maps by subtracting each single-condition map from the functional map for the same orientation but the opposite direction of motion. Examination of these maps provided unequivocal evidence for neuronal clustering according to direction. Examples obtained from two different cats are illustrated in Figures 3,4 .

Figure $3, A$ and $B$, presents the single-condition maps for opposite directions obtained from the same cortical area. Figure $3 C$ presents their difference. Dark patches represent cortical areas that were activated by upward motion. Similarly, white patches present cortical areas that were activated by downward motion. The patchy mosaic of dark and bright patches indicates that clustering of neurons according to preference of direction does exist. The map in Figure $3 C$ was scaled to the full range of gray levels. Figure $3 D$ is the same functional direction map; however, it uses the same scale of gray levels as that used for the singlecondition maps. The contrast in the single-condition maps was higher than that in the direction map, indicating that the amplitude of the single-condition maps was larger than the amplitude of the direction map. The ratio of the amplitude of differential orientation maps (not shown) and the amplitude of differential direction maps was $\sim 2$ in this experiment.

To exclude any possibility that the functional map for direction selectivity was caused by noise, the reproducibility of this map was checked. Figure 3, $E$ and $F$, presents the direction maps obtained from interlaced complementary blocks of data. To aid in assessing the similarity of these maps, two sets of crosses were added to the maps, in identical cortical locations. A high degree of similarity of the activity patterns was evident.

The results presented in Figure 3 were obtained from an 8 -week-old kitten. Similar results were obtained from four other kittens. Figure 4 illustrates results obtained from an adult cat. The format used for presentation is identical to that of Figure 3. The ratio of the amplitudes of the maps for orientation and direction in this experiment was $\sim 3.5$. In five other adult cats, we found an amplitude ratio in the range of 3 to 5 , whereas the same ratio in kittens was in the range of 2.0 to 2.5. Thus, the ratio of the amplitudes of orientation versus direction maps was larger in adult cats than in 8-week-old kittens.

\section{The effect of bar length in the stimulus on direction and orientation maps}

To clarify how the direction-selective response depends on the pattern of the visual stimulus, a different set of stimuli was presented in an experiment performed using another kitten. The 

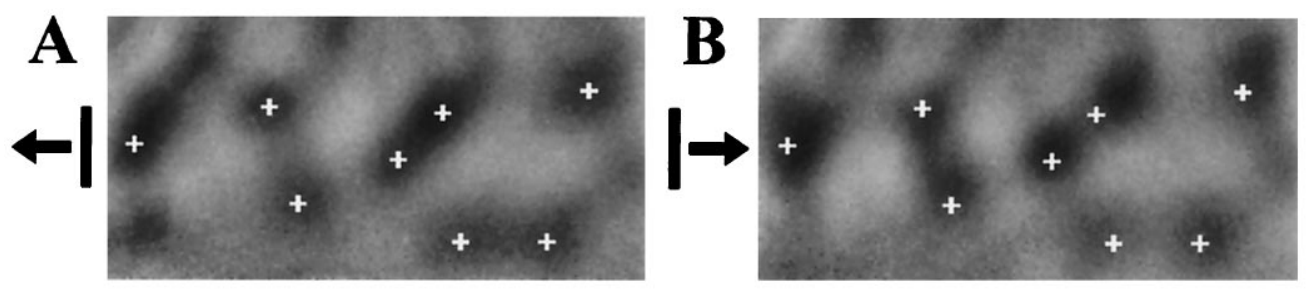

Figure 4. Clustering according to direction of motion in adult cat area 18 The results presented here were obtained from an adult cat. $A-F$ illustrate the results in a format identical to that of Figure 3. The presented maps were obtained from vertical gratings moving left and right. The whole range of gray levels represents a fractional change of $2 \times 10^{-3}$ in $A$, $B, D, 0.9 \times 10^{-3}$ in $C$, and $1 \times 10^{-3}$ in $E$ and $F$ (wavelength $630 \pm 10$ $\mathrm{nm})$. The ratio of the amplitudes here was 3.5 , and the average $\mathrm{S} / \mathrm{N}$ of the direction maps was $4.8 \pm 0.9$.
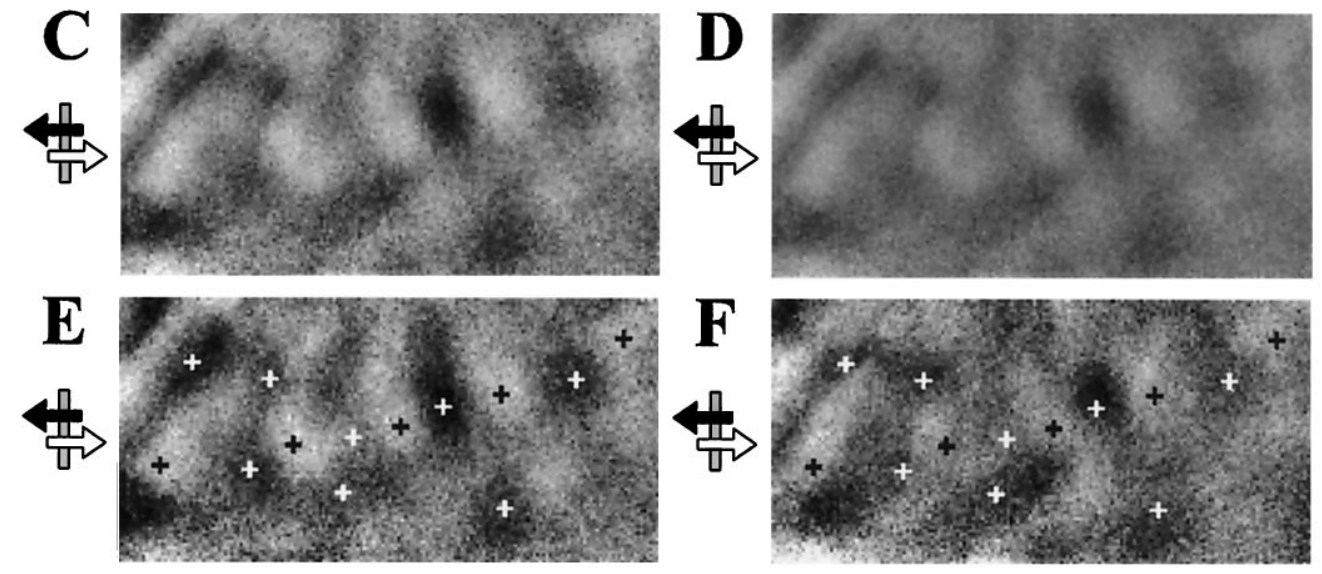

Scale bar, $1 \mathrm{~mm}$.

set of moving stimuli included five subsets displayed in the left panel of Figure 5. One of the subsets was composed of full field gratings of optimal spatial and temporal frequencies. Each of the other subsets was composed of randomly located bars. The length of the bars varied from the white squares stimulus (edge size $1.85 \times 1.85^{\circ}$, equal to the white portion of a cycle in the grating pattern) in the first subset to bars (size $1.85 \times 3.7^{\circ}$ ) in the second subset. The length was doubled again for each of the next two subsets. The average luminance of all patterns was kept constant. Each of these patterns was moved in one of four directions during different presentations $\left(0,90,180\right.$, or $270^{\circ}$; the orientation of the gratings and the bars was orthogonal to the direction). Figure 5 illustrates the differential maps for direction and for orientation obtained by stimulating with these different patterns. The top row of images corresponds to the random squares pattern, and successive rows correspond to bars whose length is doubled from one row to the next. The first column presents the differential direction maps obtained by upward versus downward motion. The second column corresponds to leftward versus rightward motion. Comparison of the spatial patterns presented across the first column indicates that the patterns of direction-selective responses were similar. The magnitudes of the direction-selective responses were comparable as well. The same features of similarity were observed for leftward versus rightward motion (second column).

The third column of functional maps shows the corresponding differential orientation maps. The sum of responses to leftward and rightward motion was divided by the sum of responses to upward and downward motion. Only weak patches were apparent in the maps obtained by the short bars. The amplitudes of the maps increased progressively as the bars became longer. This result could not be explained by low activation of the cortex by the short bar stimuli, as was determined by inspecting the global activation of cortex; the activation of cortex in fact decreased with increasing bar length of the stimulus. Therefore, we concluded that it was indeed the selective response to orientation that had a rather small amplitude for the short bars.

Overall, the selective response to direction was largely invariant to the different patterns used, in terms of both the pattern of activity and the amplitude of the direction patches. The pattern of the selective response to orientation was invariant to the different patterns of stimuli as well; however, the magnitude of the activity selective to orientation decreased for decreasing stimulus bar length. Thus, the selective response to direction was separable from the selective response to orientation.

\section{Confirmation of the functional maps by unit recordings}

To confirm the results obtained by optical imaging, and to test the preference for direction as a function of cortical depth, "targeted electrical recording" was used. After the stage of optical imaging, multiunit activity was recorded during penetrations perpendicular to the cortical surface. The locations of the penetrations were guided carefully by the maps of preference for direction. Figure 6 , $A$ and $B$, illustrates the maps used for selecting locations for the electrode penetrations. Figure $6 F$ illustrates the image of the cortical surface and the fine vascular pattern that was used to target the vertical penetrations. Figure $6 A$ illustrates the differential direction map for upward versus downward motion. Figure $6 B$ illustrates the direction map for leftward versus rightward motion. These maps were obtained from the experiment presented in the previous section. They are the mean direction maps, which were obtained by summing the corresponding maps presented in Figure 5 over all presented stimulus patterns to improve the signal-to-noise ratio. Crosses are superimposed on both direction maps, marking the locations of electrode penetrations. Most of the penetrations were located close to the centers of the patches selective for direction. The map of preferred angle of direction, computed by pixel-wise vectorial addition, is shown in Figure $6 C$. Results from the guided penetrations are presented in Figure $6 D$. The first 11 panels illustrate the results of penetrations guided by the direction map of upward versus downward motion. The last six frames illustrate the results of penetrations guided by the direction map of motion leftward versus rightward. At each depth from which data was obtained, a red line represents the preferred orientation, and a green arrow represents the preferred 

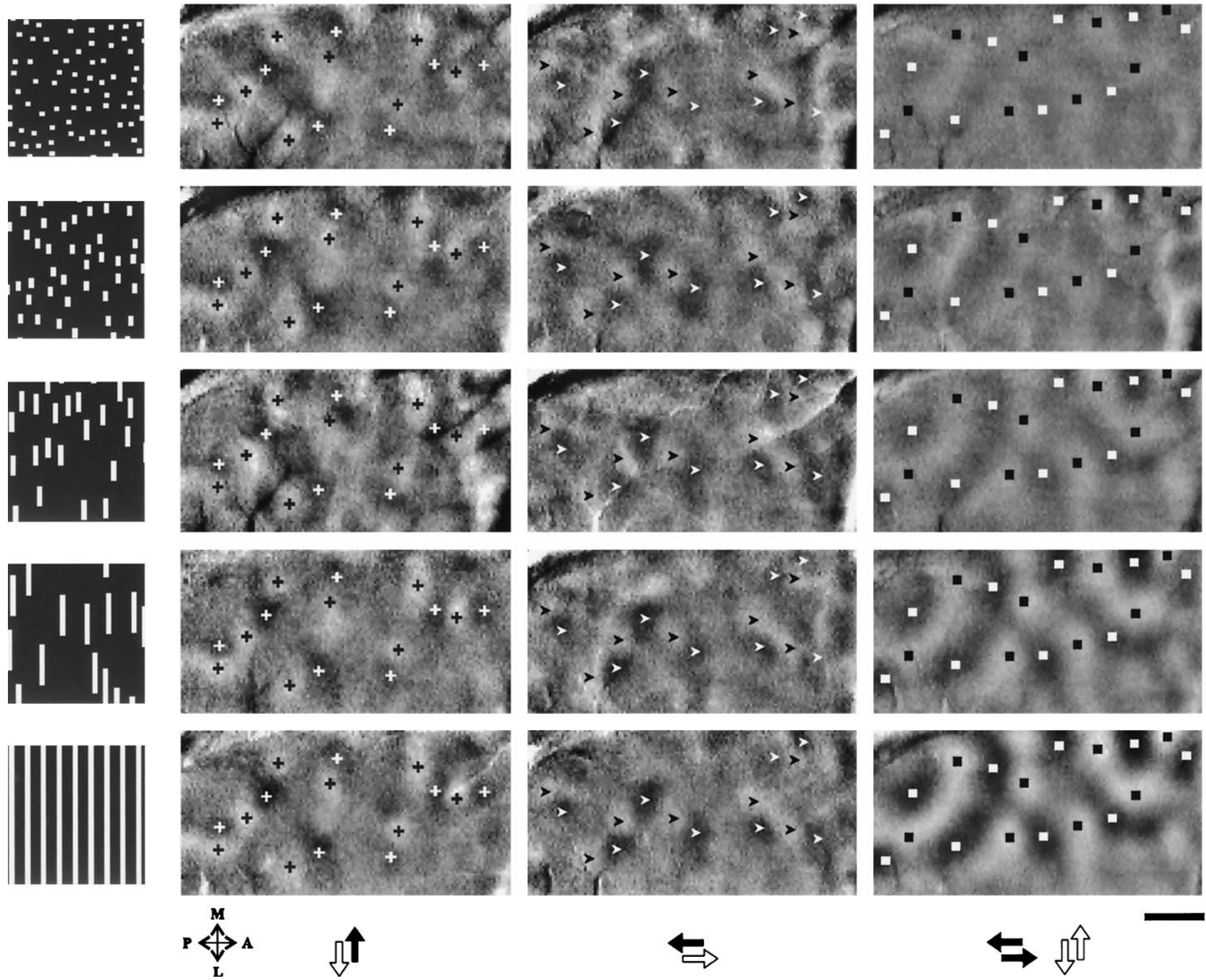

Figure 5. Direction and orientation maps evoked by various visual patterns. The set of visual stimuli included five subsets. The pattern of stimulus used to obtain the maps of each row of images is marked at the left of the row. The pattern used for the first row of images was composed of randomly located squares whose size was $1.85 \times 1.85^{\circ}$. In the next three rows, the length of the randomly located bars was doubled from one row to the next. The width of the elements was not changed. In the fifth row, full-screen rectangular gratings were used. The spatial frequency of the gratings was 0.18 cycles per degree; one cycle was divided into $33 \%\left(1.85^{\circ}\right)$ white and $67 \%\left(3.7^{\circ}\right)$ black. Each pattern was moved in one of four directions orthogonal to the orientation of bars during different presentations. The left column of images presents the differential direction maps obtained by upward versus downward motion. The middle column presents data obtained by leftward versus rightward motion. The right column presents the "orientation" maps obtained by dividing the sum of responses to leftward and rightward motion by the sum of responses to upward and downward motion. Within each column, marks are placed at identical locations. The full scale of gray levels corresponds to $5.4 \times 10^{-4}$ for the direction maps and to $7.2 \times 10^{-4}$ for the orientation maps (wavelength $630 \pm 10 \mathrm{~nm}$ ). The selective response to direction was virtually invariant to the different stimuli used, in terms of both the activity pattern and its magnitude. The pattern of the selective response of orientation was invariant to the different stimuli as well; however, the magnitude of the activity selective to orientation increased for increasing the length of the bars. Roughly, the amplitude of the maps changed in a logarithmic manner for increasing the length of the bars. $A$, Anterior; $P$, posterior; $M$, medial; $L$, lateral. Scale bar, $1 \mathrm{~mm}$.

direction. The lengths of both lines and arrows are proportional to the normalized magnitude of selective response for orientation and direction, respectively. In most of the penetrations, the preferred direction was relatively constant. An exception is shown in panel 1, where the preferred direction reversed at $\sim 750 \mu \mathrm{m}$. Local reversals also occurred in penetrations 8 and 11; however these were exceptions. The majority of penetrations tended to have a constant preferred direction.

The preferred direction detected by optical imaging at the locations of electrode penetrations is presented above each frame, indicated by the colored arrows. To compare the preferred direction of the population detected by optical imaging to that detected by multiunit recording, the mean normalized response of the units at the upper $1000 \mu \mathrm{m}$ was computed for each column. In all penetrations, the preferred direction was similar to the preferred direction detected by optical imaging. Only small deviations were observed; the largest one can be seen in panel 4 . The mean absolute deviation was $15.7 \pm 15^{\circ}$. Two factors may have contrib- 

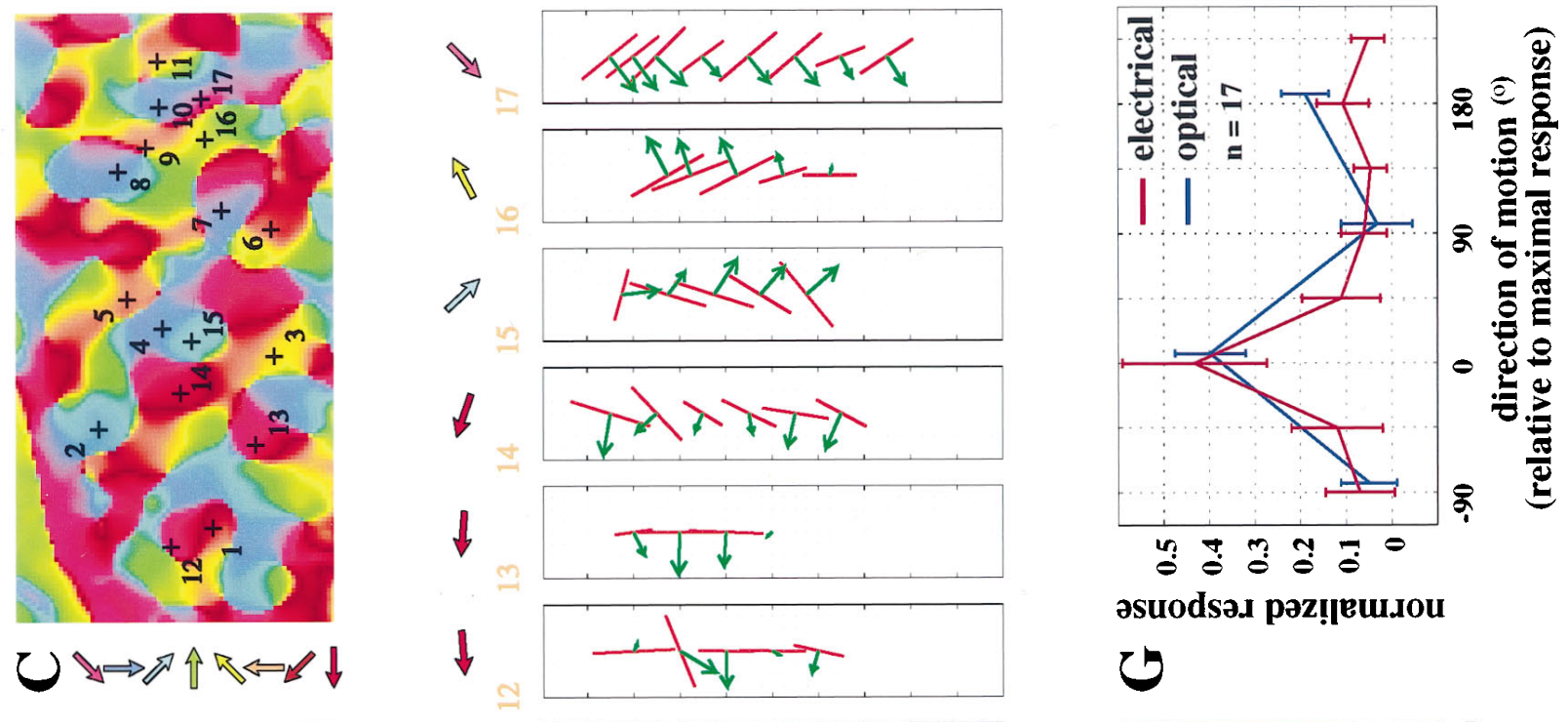

\section{๖}
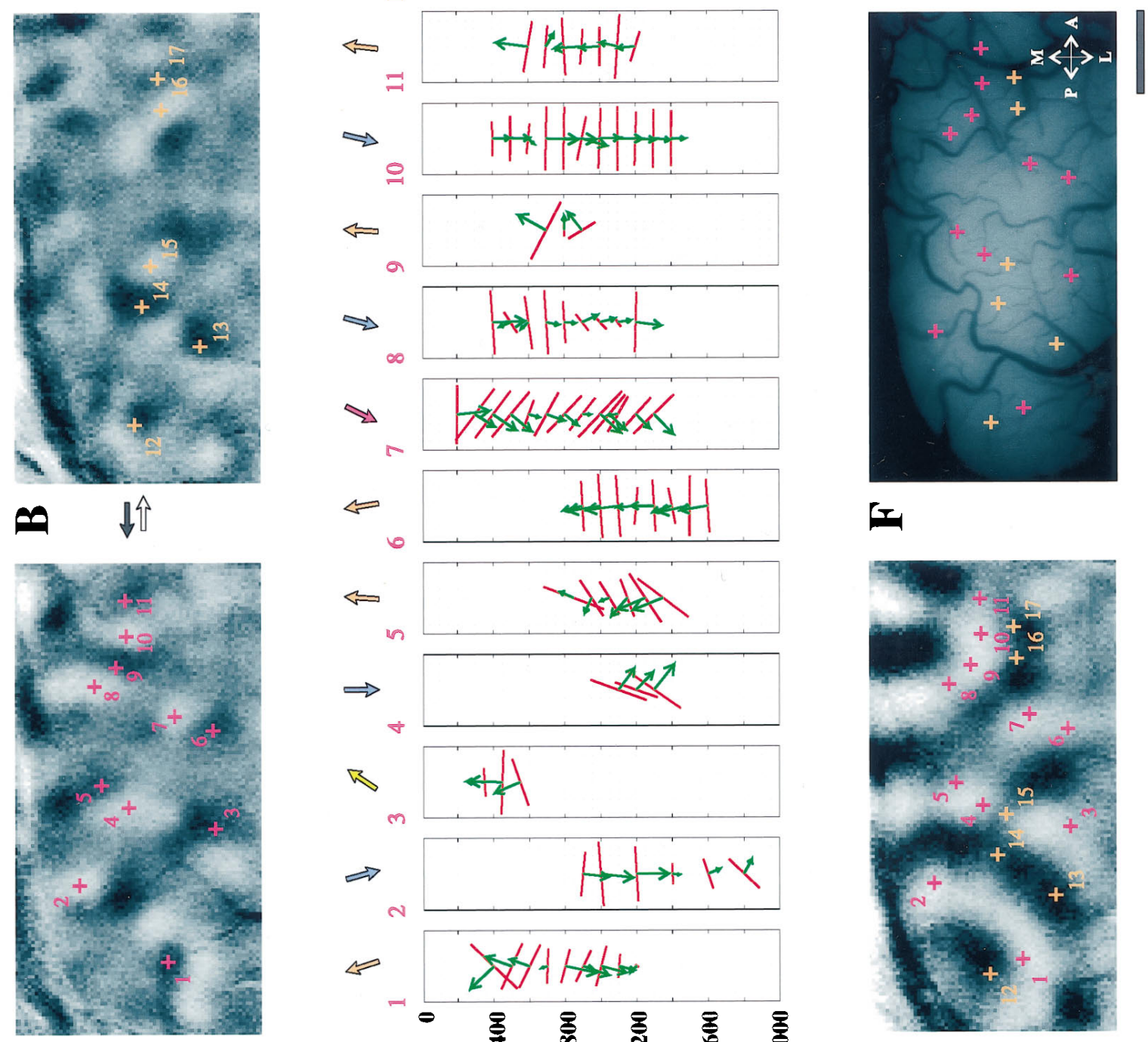

$\ll \rightleftarrows$
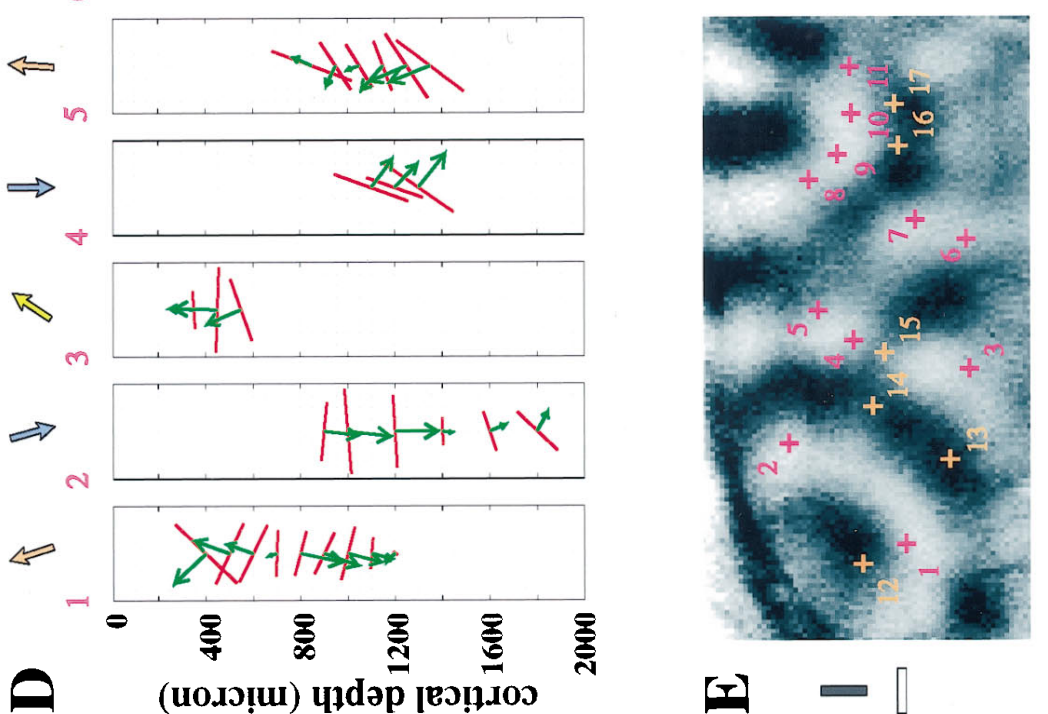

(x) $=$ [ 
uted to this small deviation. First, the set of stimuli used for calculating the angle map included only four directions: up, down, left, and right. Thus, the preferred direction detected by means of optical imaging may be inaccurate in columns whose preference was for oblique directions of motion. Second, a small uncertainty in the position of the tip of the electrode may have introduced errors. Considering these sources of error, the results obtained by the two rather different methods were indeed extremely similar.

Figure $6 E$ illustrates the differential orientation map obtained by the grating pattern. Black areas represent cortical locations that preferred vertical orientation. Again, crosses are superimposed, marking the locations of all penetrations performed. The preferred orientation detected by multiunit recording along any column was relatively constant (Fig. 6D). As in the case of direction, most of the preferred orientations detected by electrical recording were highly correlated with the preferred orientations detected by optical imaging, according to their locations within the black or white patches in Figure $6 E$.

To compare the selective responses for direction and orientation detected by optical imaging with the selectivity obtained by electrical recording, the following analysis was performed. For each multiunit recording presented in Figure $6 D$, the vector of normalized responses to all eight directions of motion was computed. Next, for each column the vector responses of all multiunits whose cortical depth is $<1000 \mu \mathrm{m}$ were summed. The result represents the mean response of the population comprising the upper layers of the column, as sampled in the session of electrical recording. Each of the 17 resulting vectors was cyclically shifted, such that the maximal response was aligned for all of the vectors. Next, the mean \pm SD of the aligned vectors was computed, averaging across all 17 columns. The result of this computation is illustrated by the red curve in Figure $6 G$. A similar procedure was performed for the optical imaging data obtained at the same cortical locations. At each of the 17 marked locations, the vector of four values of the single-condition responses was computed. Next, each of these vectors was cyclically shifted, such that the maximal response was aligned for all of the vectors. The mean \pm $\mathrm{SD}$ of the aligned vectors is presented by the blue curve. Evidently, except for small differences, the average tuning curves obtained by both methods were similar.

To determine how the responses measured by the optical imaging mapping signal correspond to those measured by electrical recording, a similar analysis was used. The stages of normalization

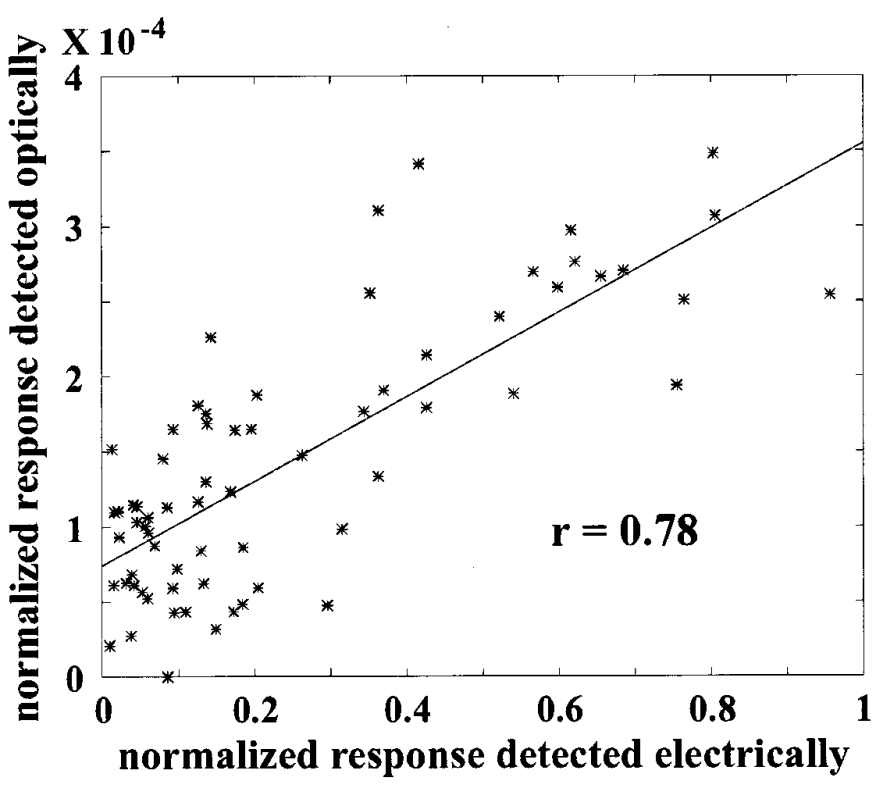

Figure 7. Relationship between the amplitude of the mapping signal and the underlying spike activity. The columnar normalized response measured by electrical recording at each site was integrated across the upper $1000 \mu \mathrm{m}$ of cortical depth. At each cortical location, the response measured by optical imaging was sampled from the corresponding singlecondition map. The 68 responses measured optically were plotted as a function of the respective responses measured by electrical recording ( $68=17$ columns $\times 4$ stimuli). The line is the best linear fit to the data obtained by linear regression. A high degree of linearity is demonstrated $(r=0.78)$.

and integration across depth were identical to those described in the previous paragraph. The 68 responses measured optically (17 columns $\times 4$ stimuli) are plotted in Figure 7 as a function of the respective responses measured by electrical recording. The line presented is the best linear fit to the data, obtained by linear regression. A high degree of linearity was demonstrated $(r=$ 0.78 ). Thus, the mapping optical signal was approximately linearly related to the sum of spikes across the upper layers of the cortex.

To assess the magnitude of direction selectivity of single cells in the center of direction-selective domains, isolated units were also recorded from two kittens. Recordings were obtained along 22 tracks perpendicular to the cortical surface. The electrode was

\footnotetext{
Figure 6. Confirmation of the optical maps by targeted unit recordings. $A$ and $B$ present the differential direction maps for upward versus downward motion and leftward versus rightward motion, respectively. These maps were used to guide vertical penetrations of an electrode for multiunit recording. Crosses are superimposed on both direction maps marking the locations of penetrations guided by each map. $C$, The map of preferred angle of direction computed by pixel-wise vectorial addition. $D$, Results from the guided penetrations. The number at the top of each separate column indicates the number of penetration, as marked in $A-C$. The vertical axis of each column is scaled to the range $0-2000 \mu$ m, representing estimated cortical depth. The preference of multiunit for orientation (red lines) and direction (green arrows) is presented as a function of cortical depth. The lengths of lines and arrows are proportional to the normalized magnitudes of selective responses for orientation and direction, respectively. The colored arrows above the columns correspond to the preferred direction detected by optical imaging. The colors correspond to the colors of the preferred direction angle map, at the location of each penetration. The mean absolute deviation of the preferred angle detected by optical imaging relative to the angle measured by electrical recording was $15.7 \pm 15^{\circ} . E$, The differential orientation map obtained by stimulating with the grating pattern. Dark and bright areas represent cortical locations that preferred vertical and horizontal orientation, respectively. The preferred orientation detected by multiunit recording along each column (as presented in $D$ ) was relatively constant. As in the case of direction, most of the preferred orientations detected by electrical recording were highly correlated with those detected by optical imaging. Note that the panels whose multiunits presented oblique preferred orientation-such as columns 4, 5, 15, and $17-$ were located as expected in gray zones between the black/white patches of the orientation map. Thus, their preference for oblique orientation is consistent with the preference detected by optical imaging. $F$, The locations of all penetrations are marked on top of the imaged cortical area. The pattern of superficial blood vessels was used to guide the electrode to the location selected for recording. $G$, The red curve represents the average columnar multiunit response of units whose depth was $<1000 \mu \mathrm{m}$. The specific columnar responses were cyclically shifted before averaging to be aligned by their maximal response. The blue curve represents the mean columnar response obtained by optical imaging, at the locations of electrode penetrations (the two curves were normalized).
} 


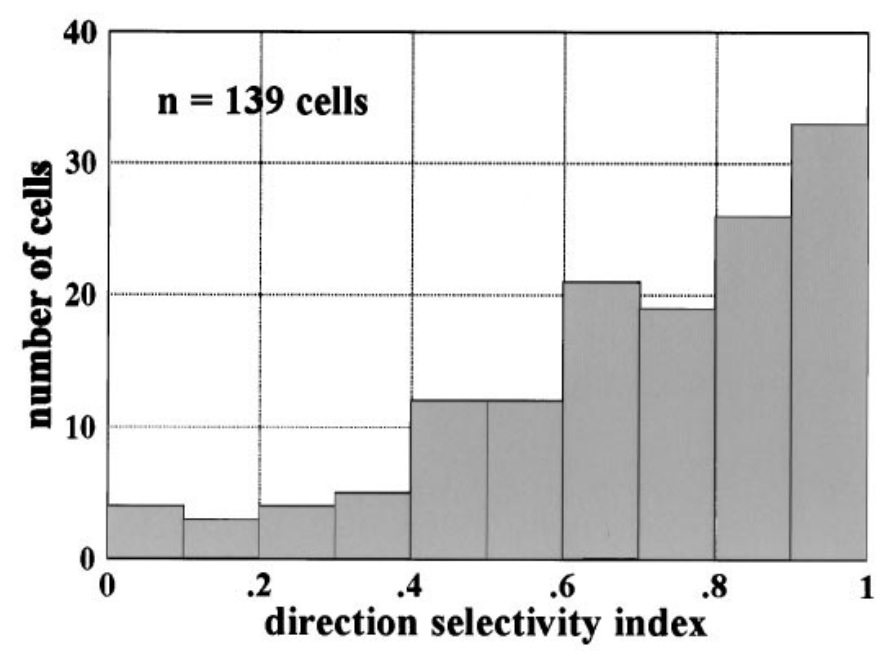

Figure 8. Direction selectivity of single neurons at the center of directionselective domains. One hundred thirty-nine isolated units were recorded from two kittens. All recordings were performed along tracks perpendicular to the cortex, targeted to the center of direction-selective domains. The sum of spikes of each cell during the presentation of each condition was computed. The direction that evoked the maximal response was referred to as the preferred direction $(P D)$. The direction index $(D I)$ was defined as $D I=\left(\right.$ response to $(P D)-$ response to $\left.\left(P D+180^{\circ}\right)\right) /$ response to $(P D)$. The distribution of direction indices is illustrated. It is skewed toward high values of direction selectivity. Eighty-one percent of the cells exhibited a direction index $\geq 0.5$.

targeted to the center of direction-selective patches, as described above. Figure 8 illustrates the distribution of direction indices of 139 of the recorded cells (see Figure legend). The cells that were included in the analysis exhibited average firing rate $\geq 0.5 \mathrm{~Hz}$ during the movement of all stimuli (total time of motion of all stimuli was in the range of 100 to $200 \mathrm{sec}$ ). The distribution was clearly skewed toward high values of direction indices: $81 \%$ of the neurons exhibited direction-selectivity index $>0.5$.

\section{Direction maps are independent of contrast and temporal frequency}

To determine whether the spatial pattern of population activity as a function of direction is sensitive to the contrast of the stimulus, a set of vertical and horizontal gratings was used. The stimuli were moved in four directions of motion. For each direction used, three different contrasts were presented: $0.2,0.4$, and 0.8 . The pattern of direction-selective maps was independent of the contrast used (not shown); however, the amplitude of the maps increased for increasing contrast.

To determine whether the pattern of population activity as a function of direction is sensitive to the temporal frequency of the stimulus, another set of vertical and horizontal gratings was used. The stimuli were moved in four directions of motion. For each direction used, two different temporal frequencies were presented: 3 and $6 \mathrm{~Hz}$. The pattern of direction-selective maps was independent of the temporal frequency used (not shown); however, the amplitude of the maps was higher for the higher temporal frequency used.

\section{Continuity of direction maps as a function of the direction of motion}

Direction-selectivity maps were obtained from a set of eight gratings moving in 16 directions. Figure 9 illustrates the eight differential direction maps obtained for all tested directions of motion. The orientation of the presented stimuli and their directions of motion are marked at the bottom right corner of the maps.

Three types of cortical activation regions are evident in these differential maps. Dark areas exhibited preference for one direction (marked by the black arrows). Bright patches exhibited preference for the opposite direction (white arrows). Approximately $73 \%$ of pixels from all combined images were occupied by gray level values that were within one quarter of the whole range of gray levels, centered around the mean gray level. The responses within these cortical regions to both directions were approximately equal.

Pairs of neighboring images represent direction maps whose axis of motion differs by $22.5^{\circ}$ (the term "axis of motion" is used here for the orientation of the line parallel to the two directions used for the differential direction map). The patterns of black and white patches of images of such adjacent pairs were similar to one another. This observation suggested that the pattern of population activity was continuous as a function of the axis of motion. Pairs of opposite images along the two sides of the central plot represent direction maps whose axes of motion are orthogonal to each other. As opposed to the aforementioned similarity of neighboring images, the direction maps for orthogonal axes of motion were not similar; sets of adjacent alternating black and white patches in one map appeared to fit within corresponding locations of gray regions in the map obtained from orthogonal axis of motion.

To quantitatively compare different direction maps, we computed the correlation coefficient of all possible pairs. The results are summarized by the plot in the center of Figure 9. The correlation coefficient of every direction map with every other direction map is averaged over all directions. The horizontal axis represents the difference in the axis of motion between the compared maps. The similarity of maps corresponding to axes of motion that differed by $22.5^{\circ}$ scored an average correlation coefficient of 0.43 . The correlation coefficient decreased for maps obtained from axes of motion whose difference was increased. The average correlation coefficient for direction maps whose axes of motion were orthogonal was $\sim 0$. This value implied a poor linear relationship between the direction maps corresponding to orthogonal axes of motion.

The average correlation coefficient for functional maps obtained from axes of motion whose difference was $>90^{\circ}$ was positive; it increased as the difference increased. This result was expected, because the two corresponding axes of motion actually got closer. The actual mean value for difference in the axes of motion of $\alpha^{\circ}$ was equal to the mean value related to difference of $(180-\alpha)^{\circ}$, as expected from the computation.

Overall, the quantitative comparison confirmed the aforementioned similarity of maps corresponding to similar axes of motion. We therefore conclude that the pattern of selective cortical activity is continuous as a function of the axis of motion.

\section{The overall organization of direction selectivity}

The previous section demonstrated differential maps for direction for all tested axes of motion. Next, we were interested in the overall topography of the representation of direction along the cortical surface. A complete set of single-condition maps was combined by pixel-wise vectorial analysis. The result of this analysis was a vectorial representation of preferred directions across the cortical surface, including the magnitude of the preference. Each location in Figure $10 A$ is color-coded for the dominant, preferred direction of the underlying cortical area. Arrows are 

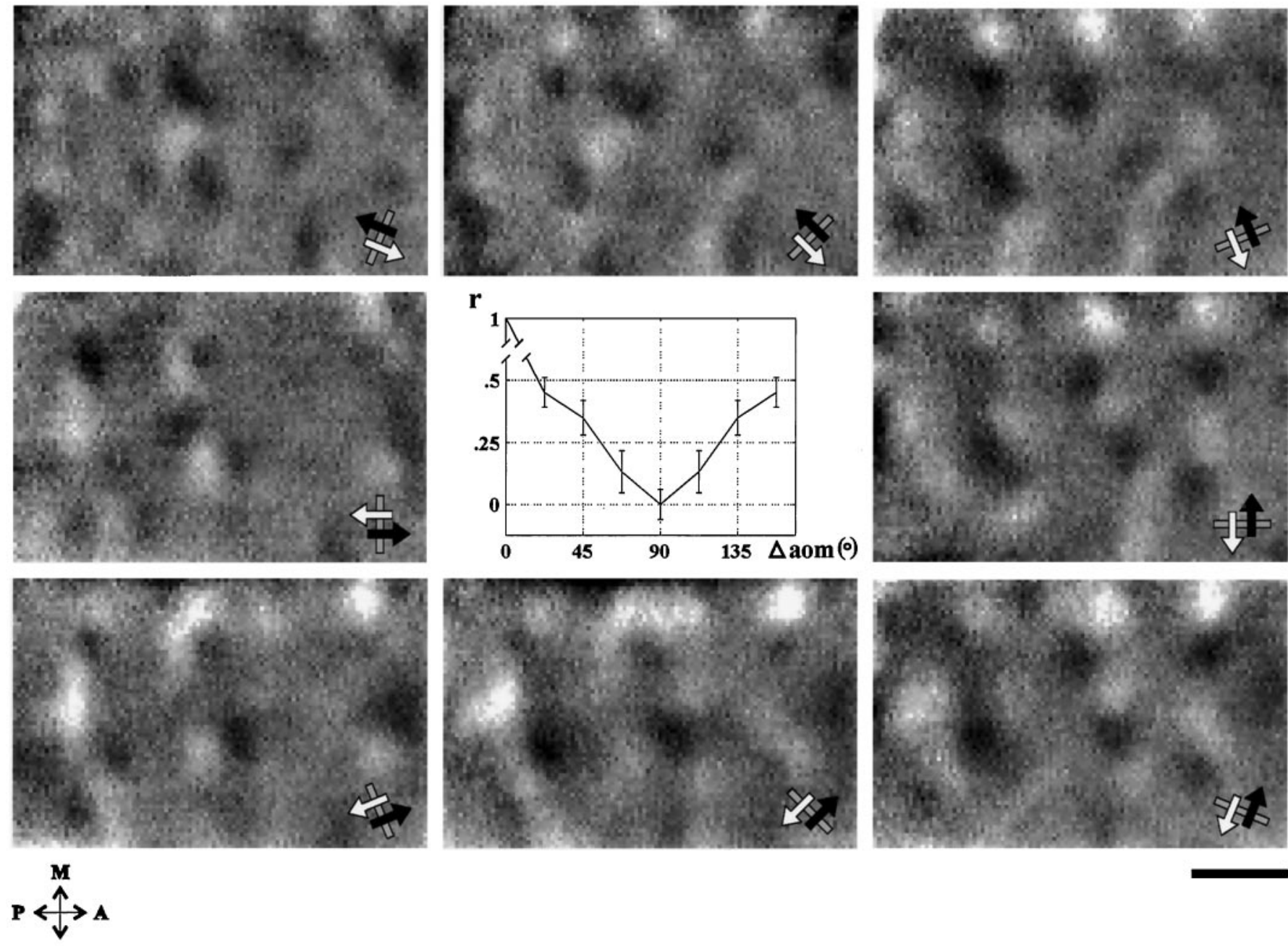

$\mathbf{L}$

Figure 9. A full set of differential direction maps. Eight different gratings, each moving in one of two opposite directions, were presented to the cat while activity maps were collected. The set of eight differential direction maps is presented. Dark and bright patches in each image represent cortical areas that preferred motion in the direction of the black and white arrows marked on the image, respectively. The entire range of gray levels represents a fractional change of $3.6 \times 10^{-4}$ (wavelength $650 \pm 10 \mathrm{~nm}$ ). The image of the corresponding cortical surface and superficial blood vessels is presented in the center of Figure 1. The maps contain significant portions of gray areas, implying no preference for any of the two directions of motion. Pairs of differential direction maps produced by gratings of similar orientations (adjacent images in the figure) were similar (the middle image in the left column should be compared to the image above it only after reversing the white and black patches in one of the images, because the color look-up table used here is not cyclical). The similarity between direction maps decreased as the difference in the corresponding axes of motion increased. These trends of similarity between differential direction maps are quantified by the plot in the center. The comparison of maps to one another was performed by calculating the correlation coefficient between corresponding pixels. The average correlation coefficient between pairs of direction maps is presented as a function of the angular difference in axis of motion. Direction maps that corresponded to similar axes of motion were similar $(r=0.43)$. Direction maps that corresponded to orthogonal axes of motion were uncorrelated $(r=0.00)$. $\Delta a$ om, Difference in the axis of motion. $A$, Anterior; $P$, posterior; $M$, medial; $L$, lateral. Scale bar, $1 \mathrm{~mm}$.

superimposed on top of the color-coded map to represent the preferred direction and the magnitude of preference (length of the arrow). The map reveals that the pattern of preferred direction was spatially continuous in most of the imaged cortical area. This observation is supported by the numerous adjacent patches that are of colors representing similar directions of motion. Pairs of these adjacent colors include pink-purple, cyan-green, greenyellow, and others.

On the other hand, discontinuities in the direction map were also detected, as shown in Figure $10 \mathrm{~B}$. The rate of change of the preferred direction along the cortex is illustrated. This map was computed using a gradient transform. Bright areas represent areas where a high rate of change in the preferred direction was exhibited. The bright areas in the form of lines correspond here to lines across which preferred direction reversed or nearly reversed. Areas exhibiting similar preferred direction were organized in patches. These patches were separated by lines across which the preferred direction reversed; however, they were not completely bounded by the lines. Transitions from a preferred direction to one orthogonal, or even opposite to it, were encountered along tracks where slow changes in preferred direction were exhibited without crossing a discontinuity line.

The magnitude of direction preference along the surface of the cortex is shown in Figure $10 C$. The brightness of any pixel is proportional to the length of the vectorial sum of responses of the underlying cortical area to all of the different directions of motion. 


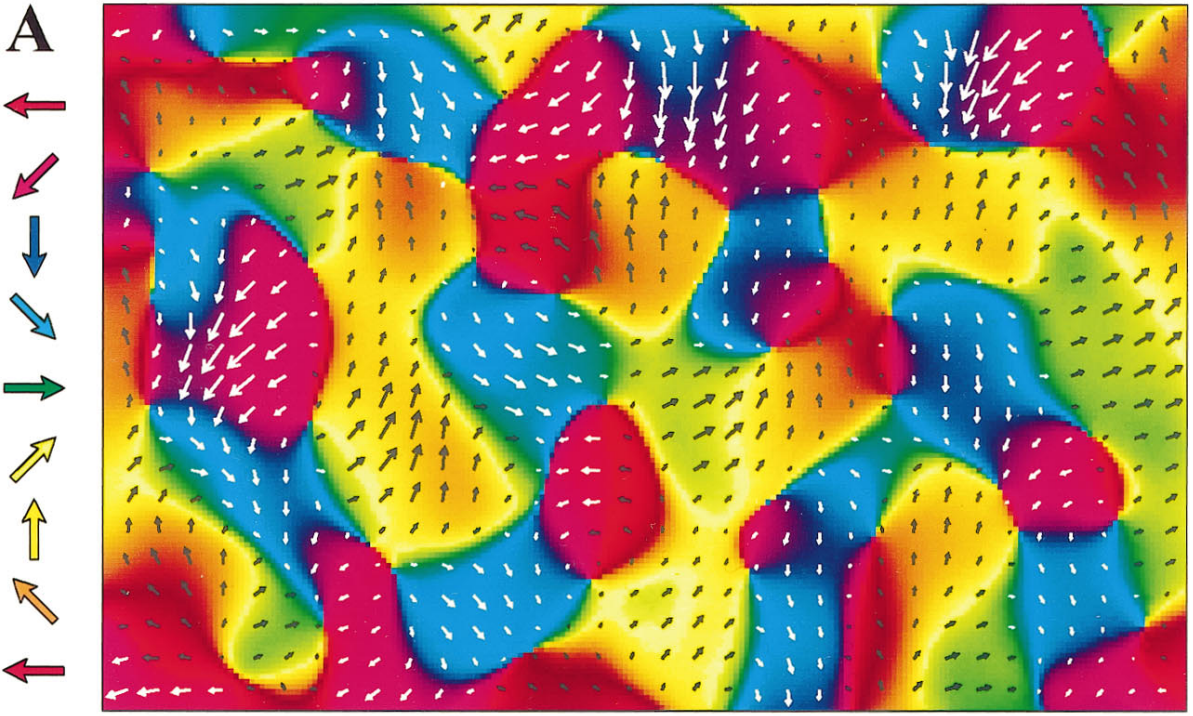

Figure 10. The overall organization for direction. A complete set of single-condition maps was combined by pixel-wise vectorial addition. $A$, The angle of the preferred direction at any location is color-coded according to the color code presented on the left. Superimposed are vectors that represent the local preferred direction (direction of the arrow) and the magnitude of preference (length of the arrow). The preferred direction was spatially continuous along the surface of the cortex in most of the imaged cortical area. $B$, The rate of change of the preferred direction. The map was computed using the gradient transform (bright denotes high rate of change). The bright areas in the form of lines here correspond to lines across which preferred direction reversed or nearly reversed. The green arrows represent the angle and magnitude of the preference of the local population. $C$, The magnitude of the direction selectivity is coded with a gray level scale. This map represents the length of the pixel-wise vectorial sum of the singlecondition maps. Bright regions correspond to high direction selectivity and dark regions correspond to low direction selectivity. Apart from the lines of low magnitude of preference, most of the cortical surface here exhibited clustering according to direction. Scale bar, $1 \mathrm{~mm}$.
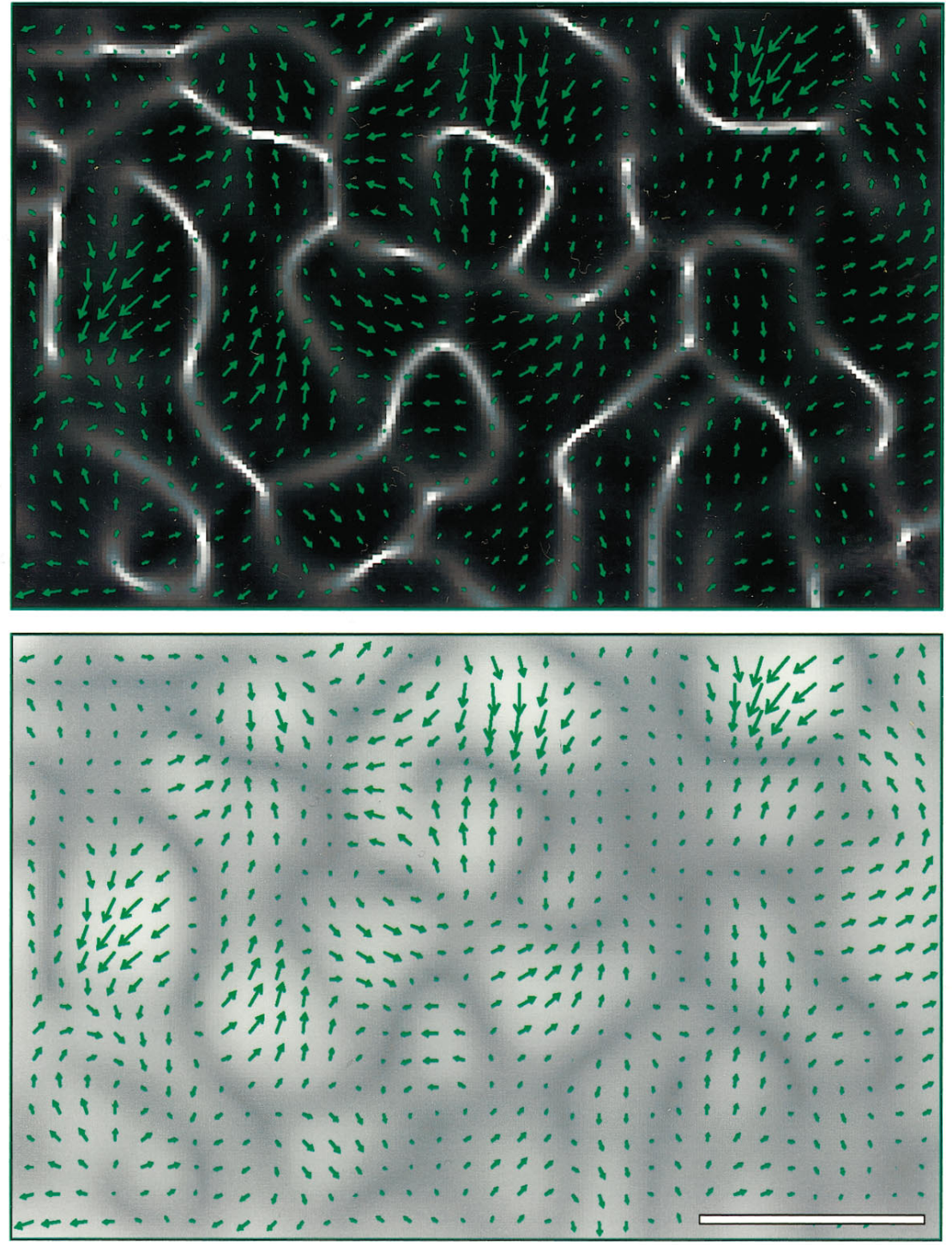
Bright patches represent areas whose selectivity for direction was pronounced. Dark areas represent a low magnitude of direction selectivity. Superimposed are arrows that represent the local preferred direction. Again, the overall pattern was composed of continuous patches of high magnitude of preference for direction. These patches were separated by domains of low preference for direction, in the form of lines. Careful examination reveals that the lines of discontinuity within the map for direction preference (Fig. 10B) nearly coincide with the lines of low magnitude in the map of the preference magnitude (Fig. 10C). In addition to the lines across which the preferred direction reverses, segments of low magnitude can be seen in areas of relatively fast changes, from a direction to one orthogonal to it.

The width of the patches that demonstrated high selectivity for direction was estimated, using a sample of distances between the centers of adjacent patches. This sample was taken from the two cats that exhibited the maps of highest degree of reproducibility. The average width of these domains was $540 \pm 125 \mu \mathrm{m}(n=30)$. This value is comparable to the previous estimation of $400-600$ $\mu \mathrm{m}$ for the most frequent separation between boundaries of continuous direction sequences (Berman et al., 1987).

Apart from the lines of relatively low magnitude of preference, the distribution of areas that presented selectivity for direction was roughly even along the surface of the cortex (Fig. 10C). Only two exceptions of areas exhibiting relatively low selectivity were observed (located in the top left and the top central part of the image). Thus, we conclude that the spatial distribution of direction-selective neurons is not limited to segregated cortical zones.

\section{Spatial frequency of direction and orientation clusters}

Figure $11 A$ illustrates the angle map of preferred orientation. Figure $11 B$ presents again the angle map of preferred direction. Although both maps use color look-up tables that offer a resolution of $45^{\circ}$, the apparent size of patches within the map for direction preference is smaller than the size of patches for orientation preference. The angle map of the orientation preference contained elongated patches whose long axis was parallel to the lateral-medial axis. The angle map of the preferred direction contained patches that were more isotropic. The ratio of the number of patches of iso-orientation to the number of patches of iso-direction was $\sim 1: 2$.

The Fourier spectra of both angle maps were analyzed. The energy in the orientation domain was somewhat anisotropic, being larger in the anterior-posterior axis, with a frequency centered around a cycle length of $1.1 \mathrm{~mm}$. The energy in the direction domain was distributed in a more isotropic manner, suggesting that no dominant axis for cyclic direction changes existed. The energy was distributed around a cycle length of $1.1 \mathrm{~mm}$, also for the direction domain.

\section{The relationship between the organization of orientation and direction}

The relationship between areas that were activated by a specific orientation and the areas that were activated by motion orthogonal to that orientation was examined. Examples are illustrated in Figure 12. The maps in the background are single-condition maps for orientation. The black patches in Figure 12 represent areas that were sensitive to the orientation designated by the black bar next to the image. The superimposed arrows represent the preferred direction and the magnitude of the selective response of the corresponding differential direction map. Fig- ure $12 A$ illustrates an instance of a patch that was tuned to a certain orientation. In terms of preference to direction, this patch was divided into two subpatches that exhibited preferences for opposite directions of motion. As was often the case, the line that separated these two subpatches crossed the orientation patch in the area of high selectivity for the specific orientation, along the center of the orientation patch. The two subpatches were located at the marginal area of the orientation-selective patch. The overall relationship was consistent with the following description. In the vicinity of an orientation-selective region, the cortical activity in response to stimulation with an oriented grating had roughly the appearance of two bell-shaped surfaces. These two surfaces corresponded to the domains that were activated by a grating moving in opposite directions. The surfaces were partially overlapping; thus their sum had the shape of one patch of activity (the orientation-selective zone). Their difference (the directionselective zones) consisted of two surfaces located at the flanks of the sum, separated from each other by a line of low selectivity. Figure $12 B$ illustrates the same relationship over a larger cortical area, using the same format. Instances of the division of single-condition patches into subpatches of direction were evident here as well. In addition, orientation patches that were fully devoted to a single direction can be seen in the right-hand part of the image.

To determine the general relationship between the domains for orientation and direction, the vectorial sums for both domains were superimposed. Figure 13 illustrates the relationship of overall preferences for direction and orientation, as exhibited in the cortices of two cats. The red lines represent the preferred orientation and the magnitude of preference by their drawn angle and length, respectively. Superimposed are green arrows, representing the preferred direction and the magnitude of the preference. The stereotyped features of the organization were the following. First, a given cortical region that exhibited orientation selectivity usually included two or more subregions of direction selectivity; second, the preferred directions of motion exhibited within these regions were approximately orthogonal to the orientation.

For a quantitative analysis of the relationship between the local preferred orientation and preferred direction, we computed the distribution of deviations from orthogonality of the two domains, using the two experiments that exhibited the highest signal-tonoise ratio. The cortical locations that exhibited low selectivity for either orientation or direction were excluded from this analysis (threshold was set at the 20th percentile for both domains). A portion of $76.5 \%$ of the analyzed cortical locations exhibited a preferred direction whose deviation from orthogonality to the preferred orientation was $<45^{\circ}$. The remaining cortical locations were not randomly located. Most locations having a deviation $>45^{\circ}$ were clustered near the endpoints of discontinuity lines in the direction domain.

\section{The relationship between the singularities in the organization of orientation and direction}

Next, we were interested in the relationship between the singularities in the maps of orientation and direction preference. The background images in Figure 13 are superpositions of the map of magnitude of direction selectivity on the maps of singularities in the orientation domain. Dark areas exhibited low magnitude of direction preference, whereas yellow areas exhibited high local rate of change in the orientation domain. Many of the singularities of orientation preference tended to be point-like (these were 
A

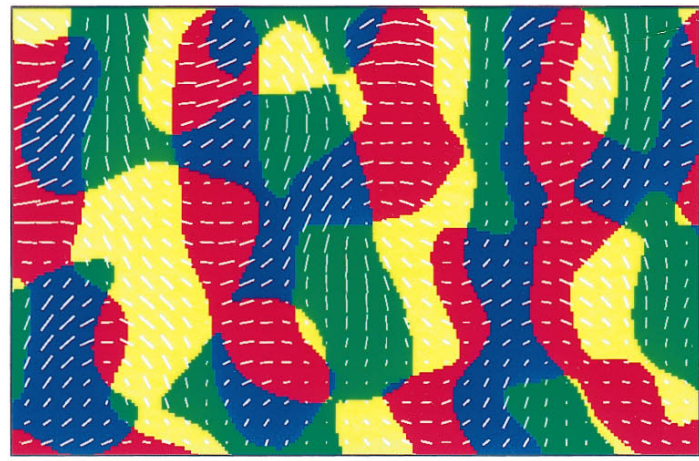

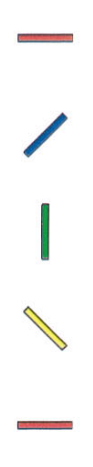
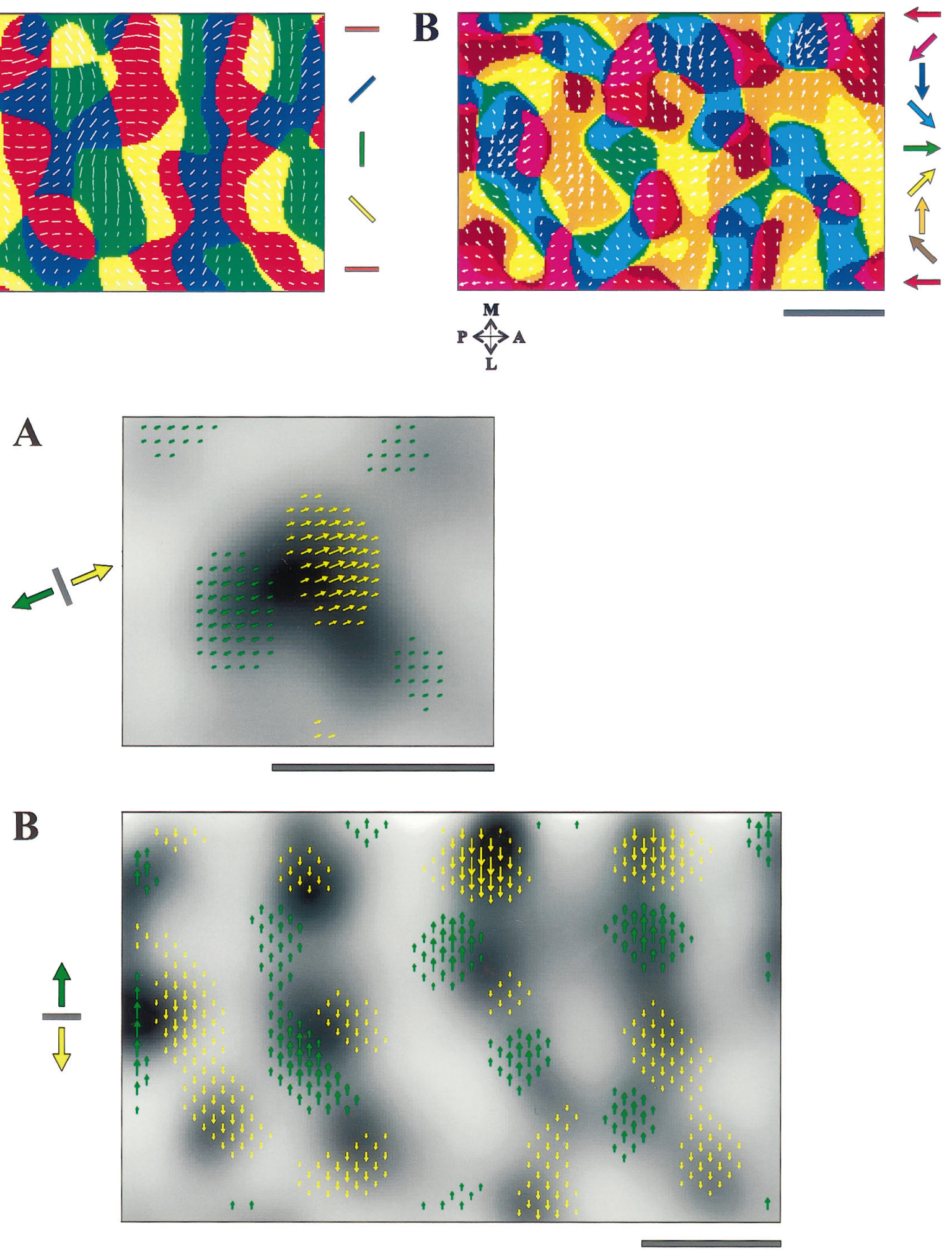

Figure 11. Top. Spatial frequencies of maps for orientation and direction preference. $A$ and $B$ present the angle maps of preferred orientation and preferred direction, respectively. Although both maps use color look-up tables that have a resolution of $45^{\circ}$, the size of patches within the map for direction preference is smaller than the size of patches for orientation preference. The ratio of the number of patches of iso-orientation and the number of patches of iso-direction presented here is $\sim 1: 2$.

Figure 12. Bottom. The relationship between orientation domains and direction clusters. The background images are single-condition maps for orientation. Black patches represent areas that responded best to the orientation designated by the black bar left of the image. The superimposed arrows represent the differential direction maps and the magnitude of preference for the appropriate axis of motion. The arrows mark the cortical area that exhibited the most prominent direction-selective response (threshold was set on the 75 th percentile in $A$, 60 th percentile in $B$ ). $A$, A patch that was 
the centers of orientation "pinwheels"). In contrast, the areas of low magnitude of direction preference were in the form of lines, as described above.

Examination of the relationships between the endpoints of lines exhibiting low magnitude of direction selectivity and the orientation singularities revealed that the dominant feature was their tendency to be connected. The "connected" type of relationship consisted of an orientation singularity with a line of direction discontinuity extending outward from it (examples are marked by ellipses). In most of the instances of this type, the direction high-gradient line tended to end in the vicinity of the orientation singularity. If the discontinuity line did not reach the singularity, the area between the discontinuity in the direction domain and the singularity of the orientation domain demonstrated a low magnitude of preference of direction. In other instances of the connected type, the direction discontinuity line ended approximately between two adjacent orientation singularities. The adjacent singularities were related to each other: they shared parts of the iso-orientation domains around them. The superimposed maps obtained from the two experiments with the highest signalto-noise ratio were examined carefully. The analyzed area from the two experiments summed up to $16.35 \mathrm{~mm}^{2}$. It contained 32 well defined orientation centers and four pairs of adjacent orientation centers as well as 59 direction discontinuity line endpoints. Of these discontinuity line endpoints, $71 \%$ were classified as belonging to the connected type and ended in an average distance of $65 \mu \mathrm{m}$ from a singularity (see Appendix for formal classification). In the other instances of endpoints (29\%), the direction discontinuity line neither ended in the vicinity of an orientation singularity nor pointed in a general direction toward an orientation singularity (examples are marked by arrows; the average distance to the closest orientation singularity in these cases was $370 \mu \mathrm{m}$ ); however, the area separating the endpoints from the singularity demonstrated both a relatively high rate of preferred direction change and a preferred direction that was not perpendicular to the preferred orientation (deviation $>45^{\circ}$ ). These organizational features seen in the separating regions may reflect a methodological artifact rather than a physiological reality; they may result from low-pass filtering of a "standard" direction discontinuity line that intersects the singularity.

Of the 36 orientation singularities, $33 \%$ exhibited no extension of a direction discontinuity of the connected type, $28 \%$ had one such extension, and $39 \%$ presented more than one extension. If we considered the nonconnected endpoints as if they were connected to a singularity through the aforementioned separating areas, the distribution of orientation singularities was $6 \%$ with no extension of a direction discontinuity, $53 \%$ with one extension, and $41 \%$ with more than one extension.

Apart from the vicinity of their endpoint, the lines of direction discontinuity tended to run through the centers of iso-orientation domains, where the magnitude of orientation preference demonstrated a ridge-like maximum. This implied that the lines of low magnitude were the result of distributed responses in the direction domain rather than low absolute activation of the cortex.

An example of the relationship between the architectures of orientation and direction preference is illustrated in Figure 14.
Two singularity points of low selectivity for orientation were very close to each other. Around the short line that connected them, two cycles of $180^{\circ}$ of preferred orientation were organized. The orientation around both singularities changed in the same direction (counterclockwise in the presented example). Superimposed on this was an organization of a full cycle of preference $\left(360^{\circ}\right)$ for direction. In the two aforementioned carefully analyzed maps, four instances of such a relationship were observed. These were instances of direction singularity endpoints of the same line, which were both connected to orientation singularities. In all four instances, the direction singularity line connected the orientation singularities in the shortest possible route.

In other instances in which the lines of discontinuity in the direction domain did not connect adjacent orientation singularities, these lines were usually curved. There was no obvious tendency for the lines to run across the cortex in a particular direction, nor was there an obvious preferred orientation at which the direction reversals would occur; the orientation often varied along the length of such lines.

\section{Organization of direction selectivity revealed by tangential electrode penetrations}

To confirm the general organization detected by optical imaging, electrical recordings were performed using tangential penetrations. The results from one of these penetrations are shown in Figure 15. The inset illustrates part of the exposed cortex, the electrode at the stage of contact with the cortex, and the 4-mmlong recording track. Multiunit activity was recorded in 80 locations spaced at $50 \mu \mathrm{m}$ intervals. The set of stimuli was identical to that described previously for optical imaging.

Figure 15 illustrates the vectorial sums of responses in the domains of orientation and direction. The following features of the organization are demonstrated. First, in general the preferred direction was orthogonal to the preferred orientation; second, preferred direction changed as a function of distance parallel to the cortex, usually in a continuous manner; and third, the continuity was occasionally interrupted by a change of $180^{\circ}$ in the preferred direction. These changes were not abrupt; rather, a short segment exhibiting low magnitude for direction selectivity separated the two locations whose preferences were opposite. Similar results were obtained in three other tangential penetrations. We therefore concluded that continuity, as well as gradual changes adjacent to region of reversals, was also exhibited by multiunit activity.

\section{DISCUSSION}

\section{Clustering according to direction of motion}

This study has demonstrated clearly that clustering according to direction of motion does exist in cat area 18; however, the degree of segregation according to direction is significantly weaker than that for orientation. The amplitude of direction maps in adult cats was three to five times weaker than that of orientation maps. In kittens, the relative amplitude of direction maps was somewhat larger, only 1.5 to 3 times weaker than that of orientation maps.

The origin of difference in the ratios of amplitudes for kittens and cats is unknown. It may be attributed to age-dependent

tuned to a certain orientation is illustrated. This patch was divided into two smaller subpatches that exhibited preferences for opposite directions of motion. The line that separated these two subpatches crossed the orientation patch in the area of high tuning for orientation. The two subpatches were located at the marginal area of the orientation-selective patch. $B$, A larger area is presented. Instances of the partitioning of the orientation patches to subpatches of direction were evident here as well. Orientation patches devoted to a single direction can be seen at the right-hand part of the image. 
functional differences, to methodological factors, or to both; this issue remains to be clarified. Altogether it was easier to obtain maps for direction in kittens than in adult cats, also owing to the better signal-to-noise ratio in kittens (Kim and Bonhoeffer, 1994).

Bonhoeffer and Grinvald (1993) compared cortical activity maps obtained by stimulation with gratings of identical orientation moving in opposite directions. They found these maps to be almost identical and suggested that cells in the upper layers of area 18 are not clustered strongly into directionality columns. These conclusions are consistent with the results of the present study. Thus, in terms of the population activity, the major clustering is according to orientation preference rather than direction preference.

In the present study, optical imaging results were confirmed by means of extensive multiunit recordings. The preferred orientation and direction measured by both methods were highly correlated (Fig. 6). Moreover, the amplitudes of the mapping optical signal and multiunit data exhibited an approximate linear relationship (Fig. 7). Thus, the ratio of amplitudes of the maps of orientation and direction, as detected by optical imaging, reflects the ratio presented by the underlying spike activity. These results also suggest that previous computations of magnitude maps indeed correspond to the underlying spike activity (Bonhoeffer and Grinvald, 1993; Malonek et al., 1994; Bonhoeffer et al., 1995).

Some of the microelectrode penetrations perpendicular to the cortical surface revealed that although the orientation preference and tuning were constant along the track, the preferred direction reversed in terms of multiunit activity (Fig. 6). The reversals in preference for direction along vertical tracks are consistent with observations described previously (Berman et al., 1987); however, we cannot rule out the possibility of having crossed lines of direction discontinuity during the penetrations in which reversals occurred.

\section{Direction selectivity of single cells}

The distribution of direction-selectivity indices of single cells (Fig. 8 ) was significantly different from those reported previously. Here, $81 \%$ of the cells exhibited direction indices $>0.5$. In contrast, previous studies (Orban et al., 1981; Berman et al., 1987) presented distributions that were rather uniform, in which $\sim 45 \%$ of the cells exhibited direction indices $\geq 0.5$. The origin of this discrepancy is not clear. Their measurements were taken from random locations in adult cat area 18, whereas the measurements in the current study were targeted to the center of directionselective domains in 8-week-old kittens. Thus, it is possible that single units are more direction selective in the kitten than in the cat. Alternatively, the higher selectivity for direction, exhibited by the population at the centers of direction-selective patches, is partially attributable to higher selectivity of single units there, and is not solely the result of clustering cells sharing the same preferred direction. Whether the direction selectivity of single cells away from the centers is reduced remains to be explored.

\section{Separability of the selective responses to orientation and to direction}

We have shown that both the spatial pattern and the amplitude of the population activity selective for direction were nearly independent of the spatial anisotropy of the stimulus used (e.g., small squares vs long bars). In contrast, the difference in spatial anisotropy of the stimulus had a large effect on the amplitude of the orientation maps. The amplitude of the orientation maps was barely measurable for the random squares and increased monotonically as a function of bar length (Fig. 5). Gizzi et al. (1990) argued that comparing responses to spots and lines is an unsatisfactory test for the classification of a single neuron as presenting "pure" direction selectivity versus direction selectivity that is secondary to its orientation selectivity. At the level of population activity explored here, it seems that random dots moving in opposite directions were adequate stimuli for obtaining pure direction maps (differential).

The functional organization of direction was highly related to that of orientation, as described in Results. Given that neurons selective for opposite directions were located within the same orientation column, one might expect that the amplitude of the direction-selective response would be correlated with the amplitude of the orientation-selective response; however, the opposite result was obtained. In particular, the short bars stimuli activated the direction patches more selectively than the orientation patches (Fig. 5). Altogether, although the functional organization of direction is related to that of orientation, the selective responses to these features of the stimulus are separable.

In addition to being independent of the aspect ratio of stimulating bars, we found that the spatial patterns of the direction maps were independent of stimulus contrast and velocity, which is consistent with previous reports (Albus, 1980; Orban et al., 1981). Also, the small squares stimuli produced direction maps of a higher amplitude than that of the corresponding orientation maps. Altogether, it seems that the functional organization for direction is an important feature of the overall organization rather than being an epiphenomenon of the clustering with respect to orientation preference.

\section{The layout of direction-selective domains}

It was shown that the spatial pattern of activity selective for direction was mostly continuous as a function of distance along the cortical surface. Fractures in the preference maps, however, did occur in the form of lines of discontinuity. The preferred directions on both sides of these lines were often opposite.

The directional selectivity shown by cortical neurons seems to be created largely by intracortical mechanisms, perhaps involving lateral inhibitory interactions across the cortical map (Sillito, 1977; Eysel et al., 1988). The layout of clustered directionality-selective cells as described here is an appropriate candidate for short lateral inhibitory connections for a cortical mechanism underlying direction selectivity.

Apart from the lines of low magnitude of preference, most of the imaged cortical surface exhibited a direction-selective response. Thus, the direction-selective cells in cat area 18 are not segregated spatially from nondirectional cells. This finding is in contrast to the spatial distribution of cells in monkey V2, where direction-selective neurons are found mainly in the thick cytochrome oxidase stripes (DeYoe and Van Essen, 1985).

\section{Relationship between the organization of orientation and direction}

The preferred direction was found to be nearly perpendicular to the preferred orientation. This relationship is not a trivial mathematical necessity: the responses to the two directions orthogonal to the preferred orientation could be strong but equal. The responses to the two directions orthogonal to a nonpreferred orientation could be relatively weak but different. Therefore, the vectorial sum of these hypothetical responses would yield a preferred direction that is not orthogonal to the preferred orienta- 

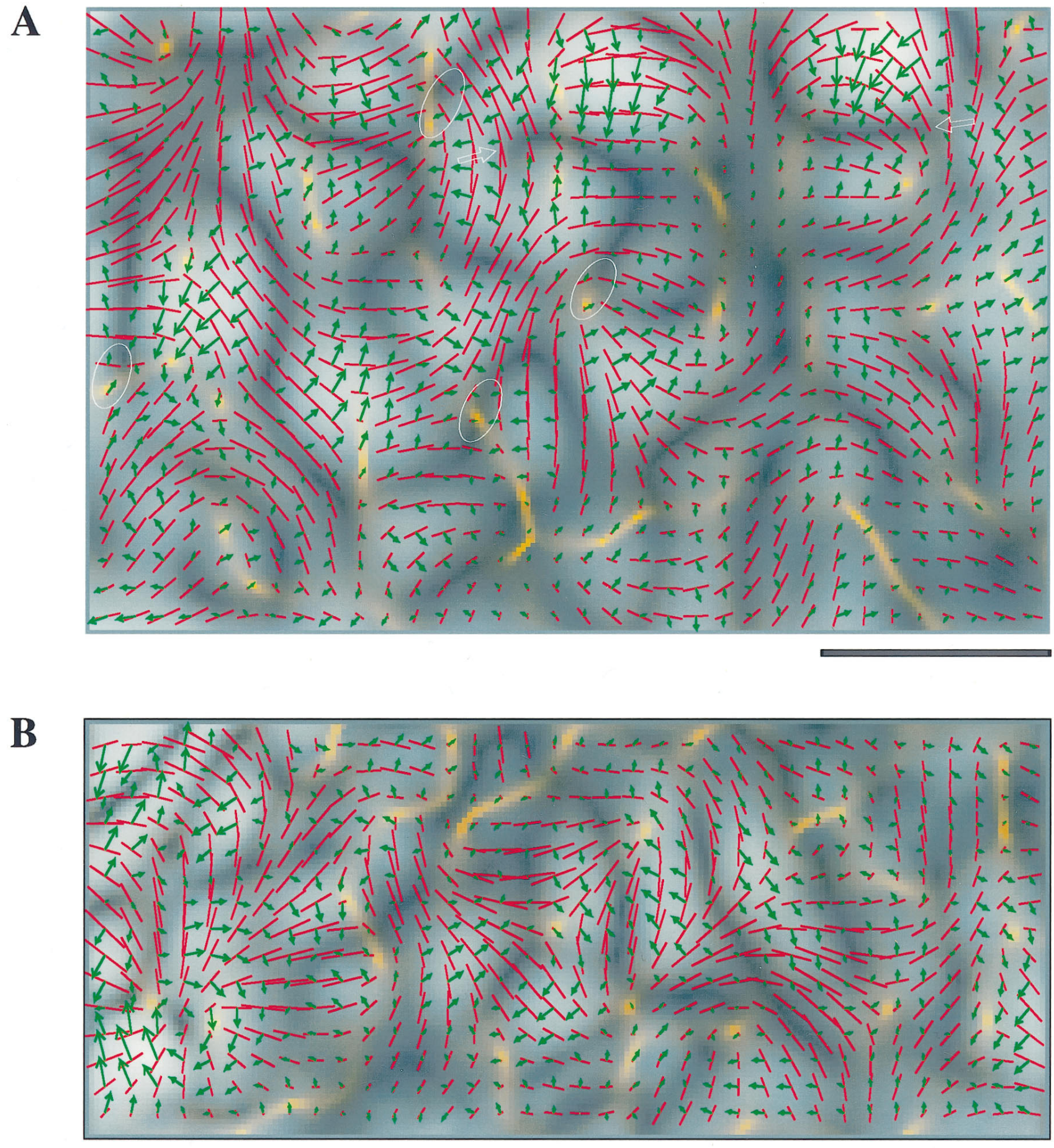

Figure 13. The overall relationship between the organization of orientation and direction. The red lines represent the preferred orientation and the magnitude of preference by their angle and length, respectively. Superimposed are green arrows, representing the local preferred direction. At the background, in gray-level presentation, is the map of magnitude for direction preference (dark represents low magnitude). Locations coded in yellow exhibited a high rate of change of preferred orientation angle (orientation singularities, computed using the gradient transform). $A$ and $B$ are examples from a kitten and an adult cat, respectively. A patch that exhibited orientation selectivity often included two or more patches of direction selectivity. The preferred directions of motion exhibited within these patches were mostly orthogonal to the orientation. Thus, the direction patches within a single orientation patch often represented preferences for opposite directions of motion. The singularities of orientation preference tended to be point-like. In contrast, the areas of low magnitude of preference for the direction domain were in the form of long curved lines. These tended to run across the center of orientation domains, in which the magnitude of preference for orientation exhibited a ridge-like maximum. Many of the orientation singularities were connected to the lines depicting regions of low directionality (examples are marked by ellipses); however, endpoints of direction discontinuity lines in locations other than orientation singularities existed (examples are marked by arrows). Scale bar, $1 \mathrm{~mm}$. 

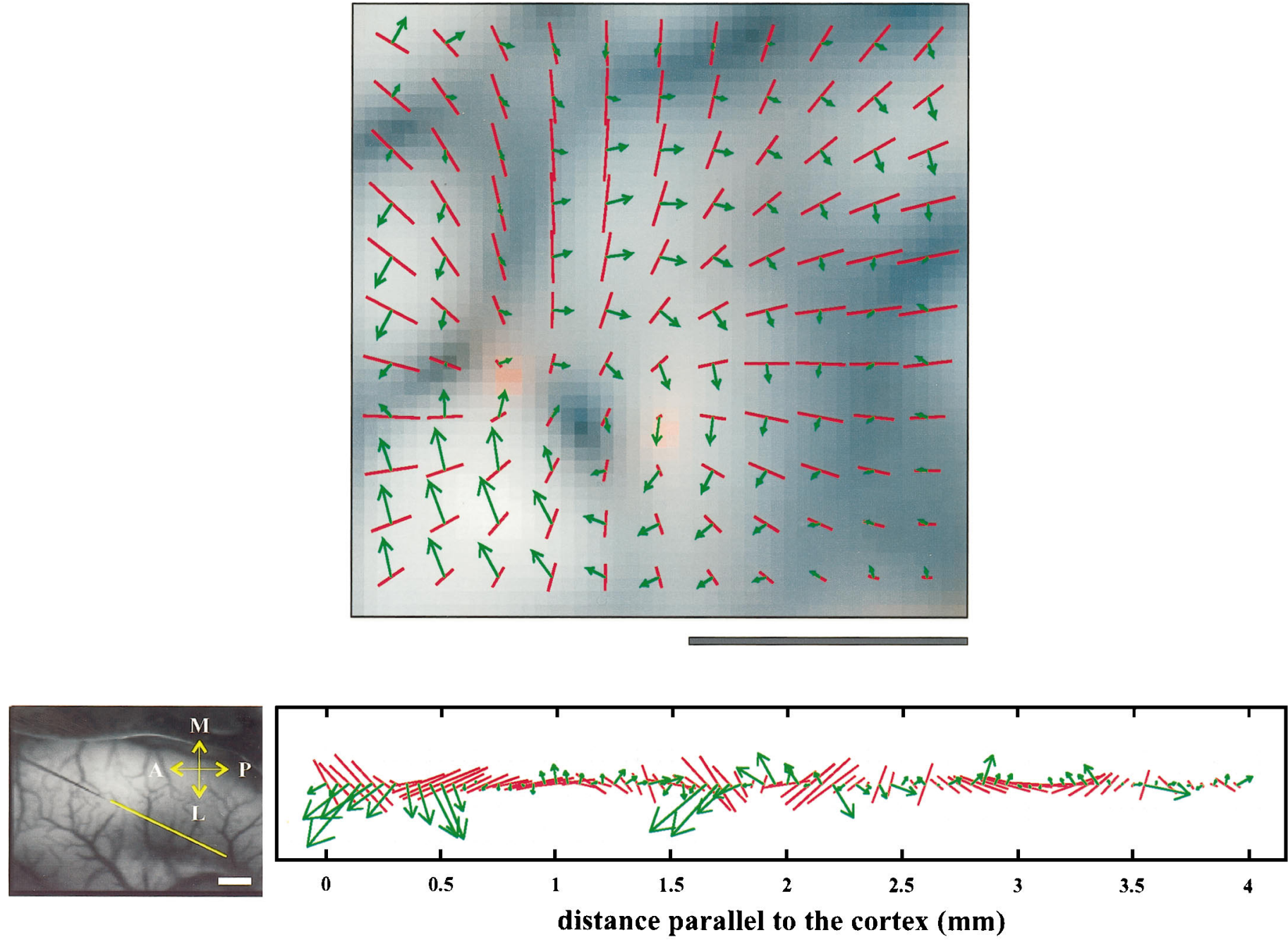

Figure 14. Top. An instance of a $360^{\circ}$ layout for direction preference. The format used here is identical to that of Figure 13 . Because the amplitude of direction selectivity is smaller than the amplitude of the orientation selectivity, the lengths of the orientation lines were scaled down by 1.5 for clarity. The orientation singularity at the right gives rise to one direction discontinuity line, whereas three such lines extend from the left singularity. Scale bar, $1 \mathrm{~mm}$.

Figure 15. Bottom. Preferences for orientation and direction examined by electrical recording along a tangential track. Multiunit data were collected in locations separated $50 \mu \mathrm{m}$ from one another. The image illustrates the exposed cortex, the electrode, and the recording track. The set of stimuli was composed of eight oriented gratings, each of which was moved in two directions. The red lines represent at each location the vectorial sum of responses to all orientations. The green arrows represent the vectorial sum of responses to all directions of motion. For clear presentation, the length of the arrows was doubled relative to that of the lines. The preferred direction changed in a continuous manner along the track, except for several locations at which reversals occurred. The magnitude of the direction preference next to reversals was low along at least $150 \mu \mathrm{m}$. Similar to the optical imaging results, the preferred direction was approximately orthogonal to the preferred orientation.

tion. Neither is this perpendicular relationship a trivial consequence of the set of stimuli we used. The use of a set of gratings stimuli whose direction is orthogonal to its orientation is justified by the similarity of direction-selective pattern of activity obtained from the random squares pattern and the gratings pattern (Fig. 5).

Because the activity of the clustered populations is tuned according to orientation, and also according to direction orthogonal to the orientation, it is a necessity that a given orientation patch either be further divided into patches exhibiting preference for opposite directions of motion or be fully devoted to one direction. Both of these possibilities were demonstrated directly (Figs. 13, 15).

The endpoints of discontinuity lines in the direction domain tend to connect to the singularities in the orientation domain (Fig. 13). This finding is consistent with both the experimental evidence and the model presented by Swindale et al. (1987). Notice that a significant portion of the endpoints in Fig. 13, even among those classified connected, is close to an orientation singularity, but do not coincide with the singularity. The same phenomenon is seen in data obtained from other species (Fig. 5B, Malonek et al., 1994; Fig. 4C, Weliky et al., 1996). This observation may reflect a methodological artifact rather than a physiological reality. The lack of rotational symmetry in the vicinity of discontinuity line endpoint makes the analysis sensitive to low-pass filtering, even that originating from the tissue light scattering alone.

Although singularities that gave rise to a single-direction discontinuity line were often encountered, a significant portion of the singularities gave rise to more than one such line $(\sim 40 \%$; see Results, Figs. 13, 14). Thus, in the vicinity of orientation singu- 
larities, the mapping of preferred direction is not always as continuous as theoretically possible.

\section{Comparison to the organization of direction and orientation in monkey area MT}

It is interesting to compare the results for cat area 18 reported here to the organization of orientation and direction in a different species and a different visual area. Clustering of neurons according to direction selectivity were detected by several groups in macaque area MT (Zeki, 1974; Baker et al., 1981; Maunsell and Van Essen, 1983; Albright et al., 1984) and in the homologous LSS areas in the cat (Spear and Baumann, 1975; Spear, 1991). The findings in macaque MT were integrated into the ice-cube model (Albright et al., 1984) of the functional organization of MT, in which columns for a common axis of motion are each divided into two columns for opposite directions.

High-resolution functional maps for direction were first obtained by means of optical imaging in Aotus (owl) monkey MT (Malonek et al., 1994). The ratio of the amplitudes of clustering according to orientation to that for direction in owl monkey MT is comparable to that reported here for adult cats (3-5). In regions where a large degree of directionality was detected, preferred direction changed smoothly across the map, except for periodic lines of discontinuity dividing regions of opposing direction preferences. The qualitative description of the features of the functional architecture for direction selectivity in cat area 18 agrees remarkably well with those of owl monkey area MT. The relationship between the mapping for the orientation domain and the direction domain are similar as well. This result is quite surprising in view of the different level of area 18 and area MT in the hierarchy of visual areas.

Such a similarity has also been observed for area 17 of the ferret (Weliky et al., 1996), in which direction preference is systematically mapped. Domains having opposite direction of motion are often paired and are located within iso-orientation domains in the primary visual cortex of the ferret.

\section{Concluding remarks}

The significant organization for direction of motion detected in this study suggests that in the cat, extensive motion analysis takes place earlier than LSS cortex. Moreover, because area 18 in the cat receives direct input from the LGN, significant motion analysis is performed at a primary processing stage in the cortex.

The similarity in the types of organization exhibited in different species and cortical areas suggests that universal principles exist for mapping the domains of direction and orientation onto the cortical surface.

\section{APPENDIX}

\section{Reproducibility estimation}

The data were divided into two sets of blocks, obtained during odd and even numbered blocks of the imaging session. Three instances of the relevant differential map were computed: one from the whole data and two others from the two half-data sets. All maps were high-pass-filtered using an isotropic Gaussian whose standard deviation was equal to $500 \mu \mathrm{m}$ of cortical distance and then were low-pass-filtered using a Gaussian of $\sigma=70 \mu \mathrm{m}$. The amplitude (AMP) of the map was determined using the map from the complete data. One percent of extreme value outliers were removed, and then the amplitude was determined as the difference between the maximum and the minimum values. The map was inspected carefully to make certain that the maximum and minimum values were located in centers of functional columns rather than in noisy areas. The root mean square (RMS) of the difference of maps obtained from the two half-data sets was computed. The signal-to-noise ratio of the map was estimated as $\mathrm{S} / \mathrm{N}=\mathrm{AMP} / \mathrm{RMS}$.

\section{Fracture analysis}

To analyze the rate of change of the preferred orientation or direction, we applied a two-dimensional gradient operator to the corresponding preference map. The formula that was used to calculate the gradient magnitude was:

$$
\begin{aligned}
|\nabla I(x, y)| & =\sqrt{\left[\frac{\partial I(x, y)}{\partial x}\right]^{2}+\left[\frac{\partial I(x, y)}{\partial y}\right]^{2}} \\
& \approx \sqrt{(I(x, y)-I(x-1, y))^{2}+(I(x, y)-I(x, y-1))^{2}},
\end{aligned}
$$

with $I(x, y)$ being the "angle map" and $x, y$ the coordinates of the single pixels in the map. The cyclical nature of the angle was considered in the computation.

\section{Relationship between singularities in the orientation and direction domains}

The map of magnitude of orientation selectivity was marked for all locations in which the magnitude was less than the value of the 10th percentile. The locations of the orientation centers were superimposed by imposing a threshold on the orientation gradient map. The map of magnitude of direction selectivity was marked for all locations in which the magnitude was less than the value of the 30th percentile. The endpoints of lines in this map were referred to as the endpoints of discontinuity lines in the direction domain. The estimation of distance between the endpoints of each line and the closest orientation singularity was performed by superimposing the maps of areas marking low magnitude for the two domains. A discontinuity line endpoint was classified as belonging to the connected type if the line pointed in a general direction toward an orientation singularity located within $150 \mu \mathrm{m}$.

\section{REFERENCES}

Albright TD, Desimone R, Gross CG (1984) Columnar organization of directionally selective cells in visual area MT of the Macaque. J Neurophysiol 51:16-31.

Albus K (1980) The detection of movement direction and the effects of contrast reversal in the cat's striate cortex. Vision Res 20:289-293.

Baker JF, Petersen SE, Newsome WT, Allman JM (1981) Visual response properties of neurons in four extrastriate visual areas of the owl monkey (Aotus trivirgatus): a quantitative comparison of medial, dorsomedial, dorsolateral and middle temporal areas. J Neurophysiol 45:397-416.

Berman NEJ, Wilkes ME, Payne BR (1987) Organization of orientation and direction selectivity in areas 17 and 18 of cat cerebral cortex. J Neurophysiol 58:676-699.

Blasdel GG, Salama G (1986) Voltage-sensitive dyes reveal a modular organization in monkey striate cortex. Nature 321:579-585.

Bonhoeffer T, Grinvald A (1991) Iso-orientation domains in cat visual cortex are arranged in pinwheel-like patterns. Nature 353:429-431.

Bonhoeffer T, Grinvald A (1993) The layout of iso-orientation domains in area 18 of cat visual cortex: optical imaging reveals a pinwheel-like organization. J Neurosci 13:4157-4180.

Bonhoeffer T, Grinvald A (1996) Optical imaging based on intrinsic signals: the methodology. In: Brain mapping: the methods (Toga AW, Mazziotta JC, eds), pp 55-97. San Diego: Academic.

Bonhoeffer T, Kim DS, Malonek D, Shoham D, Grinvald A (1995) Optical imaging of the layout of functional domains in area 17 and across the area $17 / 18$ border in cat visual cortex. Eur J Neurosci 7:1973-1988. 
DeYoe EA, Van Essen DC (1985) Segregation of efferent connections and receptive field properties in visual area $\mathrm{V} 2$ of the macaque. Nature 317:58-61.

Eysel UT, Muche T, Worgotter F (1988) Lateral interactions at direction-selective striate neurons in the cat demonstrated by local cortical inactivation. J Physiol (Lond) 399:657-675.

Frostig RD, Lieke EE, Ts'o DY, Grinvald A (1990) Cortical functional architecture and local coupling between neuronal activity and the microcirculation revealed by in vivo high resolution optical imaging of intrinsic signals. Proc Natl Acad Sci USA 87:6082-6086.

Gizzi MS, Katz E, Schumer RA, Movshon JA (1990) Selectivity for orientation and direction of motion of single neurons in cat striate and extrastriate visual cortex. J Neurophysiol 63:1529-1543.

Grinvald A, Lieke E, Frostig RD, Gilbert CD, Wiesel TN (1986) Functional architecture of cortex revealed by optical imaging of intrinsic signals. Nature 324:361-364.

Hubel DH, Wiesel TN (1962) Receptive fields, binocular interaction and functional architecture in the cat's visual cortex. J Physiol (Lond) 160:106-154.

Hubel DH, Wiesel TN (1963) Shape and arrangement of columns in cat's striate cortex. J Physiol (Lond) 165:559-568.

Hubel DH, Wiesel TN (1965) Receptive fields and functional architecture in two non-striate visual areas (18 and 19) of the cat. J Neurophysiol 28:229-289.

Hubel DH, Wiesel TN (1977) Functional architecture of macaque monkey visual cortex. Proc R Soc Lond [Biol] 198:1-59.

Kim DS, Bonhoeffer T (1994) Reverse occlusion leads to a precise restoration of orientation preference maps in visual cortex. Nature 370:370-372.

LeVay S, Nelson SB (1991) Columnar organization of the visual cortex. In: Vision and visual dysfunction, Vol 4, The neural basis of visual function (Leventhal AG, ed), pp 266-315. London: Macmillan.

Malonek D, Grinvald A (1996) The spatial and temporal relationship between cortical electrical activity and responses of the microcirculation during sensory stimulation: implications for optical, PET, and MR functional brain imaging. Science 272:551-554.

Malonek D, Tootell RBH, Grinvald A (1994) Optical imaging reveals the functional architecture of neurons processing shape and motion in owl monkey area MT. Proc R Soc Lond [Biol] 258:109-119.

Maunsell JHR, Van Essen DC (1983) Functional properties of neurons in middle temporal visual area of the macaque monkey. I. Selectivity for stimulus direction, speed and orientation. J Neurophysiol 49:1127-1147.
Mountcastle VB (1957) Modality and topographic properties of single neurons of cat's somatic sensory cortex. J Neurophysiol 20:408-434.

Orban GA, Kennedy H, Maes H (1981) Response to movement of neurons in areas 17 and 18 of the cat: direction selectivity. J Neurophysiol 45:1059-1073.

Payne BR, Berman N, Murphy EH (1980) Organization of direction preference in cat visual cortex. Brain Res 211:445-450.

Ratzlaff EH, Grinvald A (1991) A tandem-lens epifluorescence macroscope: hundred-fold brightness advantage for wide-field imaging. J Neurosci Methods 36:127-137.

Shmuel A, Arieli A, Malonek D, Grinvald A (1993) Relationship between functional organization for direction of motion and for orientation selectivity in cat area 18. Soc Neurosci Abstr 19:618.7.

Sillito AM (1977) Inhibitory processes underlying the directional specificity of simple, complex and hypercomplex cells in the cat's visual cortex. J Physiol (Lond) 271:699-720.

Spear PD (1991) Functions of extrastriate visual cortex in non-primate species. In: Vision and visual dysfunction, Vol 4, The neural basis of visual function (Leventhal AG, ed), pp 339-370. London: Macmillan.

Spear PD, Baumann TP (1975) Receptive-field characteristics of single neurons in the lateral suprasylvanian visual area of the cat. J Neurophysiol 38:1403-1420.

Swindale NV (1982) A model for the formation of orientation columns. Proc R Soc Lond [Biol] 215:211-230.

Swindale NV, Matsubara JA, Cyander MS (1987) Surface organization of orientation and direction selectivity in cat area 18. J Neurosci 7:1414-1427.

Tolhurst DJ, Dean AF, Thompson ID (1981) Preferred direction of movement as an element in the organization of cat visual cortex. Exp Brain Res 44:340-342.

Ts'o DY, Frostig RD, Lieke EE, Grinvald A (1990) Functional organization of primate visual cortex revealed by high resolution optical imaging. Science 249:417-420.

Weliky M, Bosking W, Fitzpatrick D (1996) A systematic map of direction preference in primary visual cortex. Nature 379:725-728.

Worgotter F, Eysel UT (1987) Quantitative determination of orientational and directional components in the response of visual cortical cells to moving stimuli. Biol Cybern 57:349-355.

Zeki SM (1974) Functional organization of a visual area in the posterior bank of the superior temporal sulcus of the rhesus monkey. J Physiol (Lond) 236:549-573. 